\title{
Gamma positivity of the Descent based Eulerian polynomial in positive elements of Classical Weyl Groups
}

\author{
Hiranya Kishore Dey Sivaramakrishnan Sivasubramanian \\ Department of Mathematics \\ Indian Institute of Technology, Bombay \\ Mumbai 400 076, India \\ \{hkdey, krishnan\}@math.iitb.ac.in
}

Submitted: Sep 29, 2019; Accepted: Jun 10, 2020; Published: Aug 7, 2020

(C) The authors. Released under the CC BY-ND license (International 4.0).

\begin{abstract}
The Eulerian polynomial $A_{n}(t)$ enumerating descents in $\mathfrak{S}_{n}$ is known to be gamma positive for all $n$. When enumeration is done over the type $\mathrm{B}$ and type $\mathrm{D}$ Coxeter groups, the type B and type D Eulerian polynomials are also known to be gamma positive for all $n$.

We consider $A_{n}^{+}(t)$ and $A_{n}^{-}(t)$, the polynomials which enumerate descents in the alternating group $\mathcal{A}_{n}$ and in $\mathfrak{S}_{n}-\mathcal{A}_{n}$ respectively. We show the following results about $A_{n}^{+}(t)$ and $A_{n}^{-}(t)$ : both polynomials are gamma positive iff $n \equiv 0,1(\bmod 4)$. When $n \equiv 2,3(\bmod 4)$, both polynomials are not palindromic. When $n \equiv 2(\bmod$ 4 ), we show that two gamma positive summands add up to give $A_{n}^{+}(t)$ and $A_{n}^{-}(t)$. When $n \equiv 3(\bmod 4)$, we show that three gamma positive summands add up to give both $A_{n}^{+}(t)$ and $A_{n}^{-}(t)$.

We show similar gamma positivity results about the descent based type B and type D Eulerian polynomials when enumeration is done over the positive elements in the respective Coxeter groups. We also show that the polynomials considered in this work are unimodal.
\end{abstract}

Mathematics Subject Classifications: 05A05, 05A15

\section{Introduction}

Let $f(t)=\sum_{i=0}^{n} a_{i} t^{i} \in \mathbb{Q}[t]$ be a degree $n$ univariate polynomial where $a_{i} \in \mathbb{Q}$ with $a_{n} \neq 0$. Let $r$ be the least non-negative integer such that $a_{r} \neq 0$. Define $\operatorname{len}(f)=n-r$. The polynomial $f(t)$ is said to be palindromic if $a_{r+i}=a_{n-i}$ for $0 \leqslant i \leqslant\lfloor(n-r) / 2\rfloor$. Define the center of symmetry of $f(t)$ to be $(n+r) / 2$. Note that for a palindromic polynomial $f(t)$, its center of symmetry could be half integral. 
Let $\operatorname{SymPoly}_{(n+r) / 2, r}(t)$ denote the set of palindromic univariate polynomials $f(t)$ with minimum nonzero exponent of $t$ being at least $r$ and having center of symmetry $(n+$ $r) / 2$. Let $\Gamma=\left\{t^{r+i}(1+t)^{n-r-2 i}: 0 \leqslant i \leqslant\lfloor(n-r) / 2\rfloor\right\}$. It is easy to see that if $f(t) \in \operatorname{SymPoly}_{(n+r) / 2, r}(t)$, then we can write $f(t)=\sum_{i=0}^{\lfloor(n-r) / 2\rfloor} \gamma_{n, i} t^{r+i}(1+t)^{n-r-2 i}$. The polynomial $f(t)$ is said to be gamma positive if $\gamma_{n, i} \geqslant 0$ for all $i$ (that is, if $f(t)$ has nonnegative coefficients when expressed as a linear combination of elements of $\Gamma)$. If $f(t)$ is gamma positive, we also write this as " $f(t)$ is $\gamma$-positive".

For a positive integer $n$, let $[n]=\{1,2, \ldots, n\}$. Denote by $\mathfrak{S}_{n}$ the symmetric group and let $\mathcal{A}_{n} \subseteq \mathfrak{S}_{n}$ be the alternating group on $[n]$. For $\pi=\pi_{1}, \pi_{2}, \ldots, \pi_{n} \in \mathfrak{S}_{n}$, let $\operatorname{DES}(\pi)=\left\{i \in[n-1]: \pi_{i}>\pi_{i+1}\right\}$ be its set of descents and let $\operatorname{des}(\pi)=|\operatorname{DES}(\pi)|$ be its number of descents. Let $\operatorname{asc}(\pi)=n-1-\operatorname{des}(\pi)$ denote its number of ascents. Further, let

$$
\begin{aligned}
& A_{n}(t)=\sum_{\pi \in \mathfrak{S}_{n}} t^{\operatorname{des}(\pi)} \quad \text { and } \quad A_{n}(s, t)=\sum_{\pi \in \mathfrak{S}_{n}} t^{\operatorname{des}(\pi)} s^{\operatorname{asc}(\pi)}=\sum_{i=0}^{n-1} a_{n, i} s^{n-1-i} t^{i}, \\
& A_{n}^{+}(t)=\sum_{\pi \in \mathcal{A}_{n}} t^{\operatorname{des}(\pi)} \text { and } A_{n}^{+}(s, t)=\sum_{\pi \in \mathcal{A}_{n}} t^{\operatorname{des}(\pi)} s^{\operatorname{asc}(\pi)}=\sum_{i=0}^{n-1} a_{n, i}^{+} s^{n-1-i} t^{i}, \\
& A_{n}^{-}(t)=\sum_{\pi \in\left(\mathfrak{S}_{n}-\mathcal{A}_{n}\right)} t^{\operatorname{des}(\pi)} \text { and } A_{n}^{-}(s, t)=\sum_{\pi \in\left(\mathfrak{S}_{n}-\mathcal{A}_{n}\right)} t^{\operatorname{des}(\pi)} s^{\operatorname{asc}(\pi)}=\sum_{i=0}^{n-1} a_{n, i}^{-} s^{n-1-i} t^{i} .
\end{aligned}
$$

It is well known that the Eulerian polynomials $A_{n}(t)$ are palindromic (see Graham, Knuth and Patashnik [9]). Before we move to $\gamma$-positivity of $A_{n}(t)$, we define gamma positivity of homogeneous bivariate polynomials. Most of the univariate polynomials in this work have homogeneous bivariate counterparts with the following slightly more general definition of gamma positivity. Let $f(s, t)=\sum_{i=0}^{n} a_{i} s^{n-i} t^{i}$ be a homogeneous bivariate polynomial. Define the polynomial $f(s, t)$ to be palindromic if $a_{i}=a_{n-i}$ for all $i$. More generally, we consider palindromic, degree $n$ homogenous bivariate polynomials which have $n-r$ as the highest exponent of $s$ with non-zero coefficient. Thus $a_{r} \neq 0$ and by palindromicity, $a_{n-r} \neq 0$. The polynomial $f(s, t)$ has center of symmetry $n / 2$. $f(s, t)$ is said to be bivariate $\gamma$-positive if we can write $f(s, t)=\sum_{i=0}^{\lfloor n / 2\rfloor} \gamma_{n, i}(s t)^{i}(s+t)^{n-2 i}$ with $\gamma_{n, i} \geqslant 0$ for all $i$. If $f(s, t)$ is bivariate $\gamma$-positive, then $f(t)=\left.f(s, t)\right|_{s=1}$ is clearly a univariate $\gamma$-positive polynomial with the same center of symmetry.

Foata and Schützenberger (see [7] showed $\gamma$-positivity of $A_{n}(s, t)$, the bivariate version of the Eulerian polynomial. Plugging in $s=1$ in $A_{n}(s, t)$ shows $\gamma$-positivity of $A_{n}(t)$. Later, we will sketch their proof (see Theorem 8) and make relevant modifications. Subsequently, Foata and Strehl in [8] used a group action based proof which has been termed as "valley hopping" by Shapiro, Woan and Getu [17]. The same proof shows that the Narayana polynomials are $\gamma$-positive (see Petersen's book [15, Chapter 4]).

It is a well known result of MacMahon [13] that descents and excedances are equidistributed over $\mathfrak{S}_{n}$. Several refinements of the $\gamma$-positivity of $A_{n}(t)$ are known when enumeration is done with respect to both descents $\operatorname{des}()$ and excedance exc(). For two 
statistics 2-13,31-2: $\mathfrak{S}_{n} \mapsto \mathbb{Z}_{\geqslant 0}$, Brändén [3] and Shin-and-Zeng [18, 19] have shown a $p, q$-refinement by showing that the polynomial

$$
A_{n}(p, q, t)=\sum_{\pi \in \mathfrak{S}_{n}} p^{2-13(\pi)} q^{31-2(\pi)} t^{\mathrm{des}}(\pi)=\sum_{i=0}^{\lfloor n / 2\rfloor} a_{n, i}(p, q) t^{i}(1+t)^{n-2 i}
$$

where the $a_{n, i}(p, q)$ 's are polynomials with positive coefficients. The survey [2] by Athanasiadis and the Handbook chapter [4] by Brändén contain a wealth of information related to gamma positivity of polynomials arising in combinatorics.

The polynomials $A_{n}^{+}(t)$ and $A_{n}^{-}(t)$ have been studied by Tanimoto [22] who gave recurrences for $a_{n, k}^{+}$and $a_{n, k}^{-}$, the coefficients of $t^{k}$ in these polynomials. To the best of our knowledge, there are no results on $\gamma$-positivity of $A_{n}^{+}(t)$ or $A_{n}^{-}(t)$ prior to our work. In this work, we show the following results.

Theorem 1. For $n \geqslant 1$, both $A_{n}^{+}(s, t)$ and $A_{n}^{-}(s, t)$ are $\gamma$-positive iff $n \equiv 0,1(\bmod 4)$. Further, both polynomials have the same center of symmetry.

When $n \equiv 2,3(\bmod 4)$, we show that even the univariate versions $A_{n}^{+}(t)$ and $A_{n}^{-}(t)$ are not palindromic (see Lemma 10). Hence there is no hope for their gamma positivity. We thus change the question and ask for the minimum number of gamma positive polynomials which add up to give $A_{n}^{+}(t)$. When $n \equiv 2(\bmod 4)$, we show that two gamma positive summands are sufficient. When $n \equiv 3(\bmod 4)$, the situation is more interesting. We are able to show that three gamma positive summands are sufficient. We are unable to express $A_{n}^{+}(t)$ as a sum of two gamma positive polynomials but do not have a proof that $A_{n}^{+}(t)$ cannot be expressed as a sum of two gamma positive polynomials. Identical statements can be made about $A_{n}^{-}(t)$. Our results are the following:

Theorem 2. Let $n$ be a positive integer with $n \equiv 2(\bmod 4)$. Then, $A_{n}^{+}(t)$ and $A_{n}^{-}(t)$ can be written as a sum of two gamma positive polynomials.

Theorem 3. Let $n$ be a positive integer with $n \equiv 3(\bmod 4)$. Then, $A_{n}^{+}(t)$ and $A_{n}^{-}(t)$ can be written as a sum of three gamma positive polynomials.

The previous two results are only true for the univariate polynomials $A_{n}^{+}(t)$ and $A_{n}^{-}(t)$ while Theorem 1 is true for the bivariate version $A_{n}^{+}(s, t)$ and $A_{n}^{-}(s, t)$. See Remark 23 for more on this. Theorem 1 thus gives a different refinement of Foata and Schützenberger's gamma positivity result (see Theorem 8$)$ when $n \equiv 0,1(\bmod 4)$. When $n \equiv 2,3(\bmod 4)$, Theorems 2 and 3 refine the univariate version of Theorem 8 in a different sense by giving several gamma positive summands which add to give the Eulerian polynomial $A_{n}(t)$.

We generalize our results to the case when descents are summed up over the elements with positive sign in classical Weyl groups. Let $\mathfrak{B}_{n}$ denote the group of signed permutations on $T_{n}=\{-n,-(n-1), \ldots,-1,1,2, \ldots, n\}$, that is $\sigma \in \mathfrak{B}_{n}$ consists of all permutations of $T_{n}$ that satisfy $\sigma(-i)=-\sigma(i)$ for all $i \in[n]$. As it is enough to have $\sigma(i)$ for $i \in[n]$, we denote $\sigma$ by the sequence of $n$ signed integers $\sigma(i)$. In the course of our proof, we will need to enumerate descents in $\mathfrak{B}_{n}$ with signs taken into account. Signed 
descents were enumerated in $\mathfrak{S}_{n}$ by Foata and Désarménien in [6] and by Reiner [16] for other types. This part of our work has considerable overlap with the type B result of Reiner.

Let $\mathfrak{D}_{n} \subseteq \mathfrak{B}_{n}$ denote the subset consisting of those elements of $\mathfrak{B}_{n}$ which have an even number of negative entries. As both $\mathfrak{B}_{n}$ and $\mathfrak{D}_{n}$ are Coxeter groups, they have a natural notion of descent associated to them. Similar to the classical Eulerian polynomials, we thus have the Eulerian polynomials of type B and type D. The type B Eulerian polynomial was shown to be gamma positive by Chow [5] and Petersen [14] and the type D Eulerian polynomial was shown to be gamma positive by Stembridge [21] and by Chow [5]. There is further, a natural notion of length in these groups and we extend our results when we enumerate descents in these groups but restrict summation to elements with even length. For Type B Weyl groups, we need $n \geqslant 2$ and our results depend on oddness or evenness of $n$. Here, our main results are Theorems 30 and 31. For Type D Weyl groups, our main result is Theorem 39 and is applicable when $n \geqslant 3$.

If a polynomial $f(t)$ is $\gamma$-positive, then it is unimodal but if $f(t)$ is the sum of two or three gamma positive polynomials, then its unimodality is not clear. However, in Section 9, we show that all the polynomials considered in this work are indeed unimodal. We end this paper by posing some questions and stating some of our conjectures in Section 10.

\section{Preliminaries on gamma positive polynomials}

In this section, we prove a few lemmas on gamma positivity that we use repeatedly in our work. Some of them are elementary and hence we omit their proofs. We start with the following.

Lemma 4. Let $f_{1}(s, t)$ and $f_{2}(s, t)$ be two bivariate $\gamma$-positive polynomials with respective centers of symmetry $m_{1}$ and $m_{2}$. Then, their product $f_{1}(s, t) f_{2}(s, t)$ is $\gamma$-positive with center of symmetry $m_{1}+m_{2}$.

Let $D=\frac{\partial}{\partial s}+\frac{\partial}{\partial t}$ be an operator in $\mathbb{Q}[s, t]$.

Lemma 5. Let $f(s, t)$ be a bivariate $\gamma$-positive polynomial with center of symmetry $n / 2$. Then, $g(s, t)=D f(s, t)$ is $\gamma$-positive with center of symmetry $(n-1) / 2$.

Proof. Let

$$
\begin{aligned}
f(s, t) & =\sum_{i=0}^{\lfloor n / 2\rfloor} \gamma_{n, i}(s t)^{i}(s+t)^{n-2 i} \text { with } \gamma_{n, i} \geqslant 0 . \text { Then, } \\
D f(s, t) & =\sum_{i=0}^{\lfloor n / 2\rfloor} i \gamma_{n, i}(s t)^{i-1}(s+t)^{n-2 i+1}+\sum_{i=0}^{\lfloor n / 2\rfloor} 2(n-2 i) \gamma_{n, i}(s t)^{i}(s+t)^{n-2 i-1} \\
& =\sum_{i=0}^{\lfloor(n-1) / 2\rfloor} \beta_{n, i}(s t)^{i}(s+t)^{n-2 i-1} .
\end{aligned}
$$


where $\beta_{n, i}=(i+1) \gamma_{n, i+1}+2(n-2 i) \gamma_{n, i}$. It is easy to see that each $\beta_{n, i} \geqslant 0$ and that $D f(s, t)$ has center of symmetry $(n-1) / 2$, completing the proof.

The following are easy corollaries.

Corollary 6. Let $f(s, t)$ be a bivariate $\gamma$-positive polynomial with center of symmetry $n / 2$.

1. Then, for natural numbers $\ell \leqslant n, g(s, t)=D^{\ell} f(s, t)$ is $\gamma$-positive with center of symmetry $(n-\ell) / 2$.

2. Then, $h(s, t)=(s t)^{i} f(s, t)$ is $\gamma$-positive with center of symmetry $i+n / 2$.

3. Then, $h(s, t)=(s+t) f(s, t)$ is $\gamma$-positive with center of symmetry $(n+1) / 2$.

Lemma 7. Let $f(t)$ be $\gamma$-positive with center of symmetry $n / 2$ and with len $(f(t))$ being odd. Then, $f(t)$ is the sum of two $\gamma$-positive polynomials $p_{1}(t)$ and $p_{2}(t)$ with centers of symmetry $(n-1) / 2$ and $(n+1) / 2$ respectively. Further, both $p_{1}(t)$ and $p_{2}(t)$ have even length.

Proof. Let the degree of $f(t)$ be $d$ and let $f(t)=\sum_{i=n-d}^{\lfloor n / 2\rfloor} \gamma_{i} t^{i}(1+t)^{n-2 i}$. We are given that $f(t)$ has center of symmetry $n / 2$. Thus,

$$
\begin{aligned}
f(t) & =\sum_{i}^{\lfloor n / 2\rfloor} \gamma_{i} t^{i}(1+t)^{n-2 i-1}(1+t) \\
& =\left(\sum_{i}^{\lfloor n / 2\rfloor} \gamma_{i} t^{i}(1+t)^{n-1-2 i}\right)+\left(\sum_{i}^{\lfloor n / 2\rfloor} \gamma_{i} t^{i+1}(1+t)^{n-1-2 i}\right)=p_{1}(t)+p_{2}(t) .
\end{aligned}
$$

It is easy to see that $p_{1}(t)$ and $p_{2}(t)$ are $\gamma$-positive with respective centers of symmetry $(n-1) / 2$ and $(n+1) / 2$. The argument requires the exponent $n-2 i$ to be odd to enable us to pull out a $(1+t)$ factor from each term $\gamma_{i} t^{i}(1+t)^{n-2 i}$ especially when $i=\lfloor n / 2\rfloor$. If len $(f(t))$ is odd, then $n-2 d$ will be odd and hence $n-2 i$ will be odd for all $i$. It is easy to see that $p_{1}(t)$ and $p_{2}(t)$ have even length, completing the proof.

\subsection{Gamma positivity of the Eulerian polynomial}

We sketch a proof of gamma positivity of the bivariate Eulerian polynomials $A_{n}(s, t)$. We do this as it sets up the stage for proofs of other results in this paper. The result is due to Foata and Schützenberger (see [7]) and the proof given below is a modification of the proof given by Visontai in [23]. Recall that $D=\frac{\partial}{\partial s}+\frac{\partial}{\partial t}$.

Theorem 8 (Foata and Schützenberger). With $A_{n}(s, t)$ as defined in $(1)$, we have

$$
A_{n}(s, t)=\sum_{i=0}^{\lfloor(n-1) / 2\rfloor} \gamma_{n, i}(s t)^{i}(s+t)^{n-1-2 i}
$$

where the $\gamma_{n, i}$ are nonnegative integers for all $n, i$. 
Proof. (Sketch) By induction on $n$. The base case when $n=2$ is easy to see. Foata and Schützenberger showed the following recurrence

$$
A_{n+1}(s, t)=(s+t) A_{n}(s, t)+s t D A_{n}(s, t) .
$$

This can be seen by adding the letter $(n+1)$ in the $n+1$ places of each permutation $\pi \in \mathfrak{S}_{n}$. The first term $(s+t) A_{n}(s, t)$ accounts for those permutations in $\mathfrak{S}_{n+1}$ in which the letter $(n+1)$ appears in the first or the last position. The term $s t D A_{n}(s, t)$ is the contribution of all $\pi \in \mathfrak{S}_{n+1}$ in which the letter $(n+1)$ appears in positions $r$ for $2 \leqslant r \leqslant n$.

Let $T=(s+t)+s t D$ be an operator in $\mathbb{Q}[s, t]$. By induction, $A_{n}(s, t)$ is $\gamma$-positive with center of symmetry $(n-1) / 2$. By Corollary 6 , both $(s+t) A_{n}(s, t)$ and $\operatorname{st} D A_{n}(s, t)$ are $\gamma$-positive with centers of symmetry $n / 2$. Thus their sum is also $\gamma$-positive with the same center of symmetry $n / 2$. A more detailed analysis of the above argument as done in the proof of [23, Theorem 2] gives the following recurrence:

$$
\gamma_{n+1, i}=(i+1) \gamma_{n, i}+2(n+1-2 i) \gamma_{n, i-1} .
$$

The above recurrence together with the initial conditions $\gamma_{1,0}=1$ or $\gamma_{2,0}=1, \gamma_{2,1}=0$ settles non-negativity of $\gamma_{n, i}$ for all $n, i$, completing the proof.

We will need the following corollary later on. It follows from Shin and Zeng [18, Theorem 2].

Corollary 9 (Shin and Zeng). From recurrence (6), we get that $\gamma_{n, i}$ is even for all $n \geqslant 1$ and for all $i \geqslant 1$ while $\gamma_{n, 0}=1$ for all $n$. Thus, for all $n$, the only odd gamma coefficient is $\gamma_{n, 0}$.

\section{Recurrences for $A_{n}^{+}(s, t)$ and $A_{n}^{-}(s, t)$}

For $\pi=\pi_{1} \pi_{2} \cdots \pi_{n} \in \mathfrak{S}_{n}$, define its number of inversions by $\operatorname{inv}_{A}(\pi)=\mid\{1 \leqslant i<j \leqslant n$ : $\left.\pi_{i}>\pi_{j}\right\} \mid$. We first consider palindromicity of $A_{n}^{+}(s, t)$ and $A_{n}^{-}(s, t)$.

Lemma 10. For $n \geqslant 1$, the polynomials $A_{n}^{+}(s, t)$ and $A_{n}^{-}(s, t)$ are palindromic iff $n \equiv 0,1$ $\bmod 4$.

Proof. Let $\pi=\pi_{1}, \pi_{2}, \cdots, \pi_{n} \in \mathfrak{S}_{n}$. Define $f: \mathfrak{S}_{n} \mapsto \mathfrak{S}_{n}$ by $f(\pi)=n+1-\pi_{1}, n+1-$ $\pi_{2}, \cdots, n+1-\pi_{n}$. Clearly, $f$ is a bijection. Further, $\operatorname{inv}_{A}(\pi)+\operatorname{inv}_{A}(f(\pi))=\left(\begin{array}{l}n \\ 2\end{array}\right)$ and $\operatorname{des}(\pi)+\operatorname{des}(f(\pi))=n-1$. Hence, if $\pi \in \mathcal{A}_{n}$, then $f(\pi) \in \mathcal{A}_{n}$ iff $n \equiv 0,1 \bmod 4$.

If $n \equiv 2,3 \bmod 4$, then the map $f$ flips the parity of $\operatorname{inv}(\pi)$ and hence we get $a_{n, k}^{+}=$ $a_{n, n-1-k}^{-}$. Further, $a_{n, 0}^{+}=1$ and $a_{n, n-1}^{+}=a_{n, 0}^{-}=0$ and hence $A_{n}^{+}(s, t)$ and $A_{n}^{-}(s, t)$ are not palindromic.

For $\pi \in \mathfrak{S}_{n}$ let $\pi^{\prime}$ be obtained from $\pi$ by deleting the letter $n$. Suppose $\pi \in \mathcal{A}_{n}$. Then, it is easy to see that $\pi^{\prime} \in \mathcal{A}_{n-1}$ and $\pi^{\prime} \in \mathfrak{S}_{n-1}-\mathcal{A}_{n-1}$ are both possible. An identical statement is true when we get $\pi^{\prime}$ from $\pi \in \mathfrak{S}_{n}-\mathcal{A}_{n}$. 
We will add the letter $n$ to permutations in $\mathfrak{S}_{n-1}$. We need to analyse when permutations in $\mathcal{A}_{n}$ arise from $\mathcal{A}_{n-1}$ and when they arise from $\mathfrak{S}_{n-1}-\mathcal{A}_{n-1}$ in this process. For this, we will need to keep track of the position of the letter $n$ in $\pi$. Let $\pi=\pi_{1}, \pi_{2}, \ldots, \pi_{n} \in \mathfrak{S}_{n}$. We term the left-most position before $\pi_{1}$ as the initial or 'zero'-th gap and the right-most position after $\pi_{n}$ as the final gap. For $1 \leqslant i \leqslant n$, we denote the gap between $\pi_{i}$ and $\pi_{i+1}$ as the ' $i$ '-th gap of a permutation. Define npos $(\pi)=\pi^{-1}(n)$ to be the index $i$ such that $\pi(i)=n$. We start with the following definitions.

1. $\mathcal{A}_{n}^{i}=\left\{\pi \in \mathcal{A}_{n}: \operatorname{npos}(\pi)=i+1\right\}$.

2. $\left[\mathcal{A}_{n-1} \rightarrow \mathcal{A}_{n}^{i}\right]=\left\{\pi \in \mathcal{A}_{n}^{i}\right.$ with $\left.\pi^{\prime} \in \mathcal{A}_{n-1}\right\}$.

3. $\left[\left(\mathfrak{S}_{n-1}-\mathcal{A}_{n-1}\right) \rightarrow \mathcal{A}_{n}^{i}\right]=\left\{\pi \in \mathcal{A}_{n}^{i}\right.$ with $\left.\pi^{\prime} \in\left(\mathfrak{S}_{n-1}-\mathcal{A}_{n-1}\right)\right\}$.

4. $\left(\mathfrak{S}_{n}-\mathcal{A}_{n}\right)^{i}=\left\{\pi \in \mathfrak{S}_{n}-\mathcal{A}_{n}: \operatorname{npos}(\pi)=i+1\right\}$.

5. $\left[\mathcal{A}_{n-1} \rightarrow\left(\mathfrak{S}_{n}-\mathcal{A}_{n}\right)^{i}\right]=\left\{\pi \in\left(\mathfrak{S}_{n}-\mathcal{A}_{n}\right)^{i}\right.$ with $\left.\pi^{\prime} \in \mathcal{A}_{n-1}\right\}$.

6. $\left[\left(\mathfrak{S}_{n-1}-\mathcal{A}_{n-1}\right) \rightarrow\left(\mathfrak{S}_{n}-\mathcal{A}_{n}\right)^{i}\right]=\left\{\pi \in\left(\mathfrak{S}_{n}-\mathcal{A}_{n}\right)^{i}\right.$ with $\left.\pi^{\prime} \in \mathfrak{S}_{n-1}-\mathcal{A}_{n-1}\right\}$.

The way permutations in $\mathcal{A}_{n}$ arise from elements in $\mathcal{A}_{n-1}$ and $\mathfrak{S}_{n-1}-\mathcal{A}_{n-1}$ depends on the parity of $n$ and is given by the following two lemmas. Since the proofs are simple, we omit them.

Lemma 11. For a positive integer $m$, let $n=2 m+1$. Then, $\pi \in \mathcal{A}_{n}$ arises by placing " $n$ " in either the first or the last position of elements of $\mathcal{A}_{2 m}$, or placing " $n$ " in the even gaps of elements of $\mathcal{A}_{2 m}$. This way, after insertion, " $n$ " will appear in an odd position in $\mathcal{A}_{n}$. Another way to get $\pi \in \mathcal{A}_{n}$ is by placing " $n$ " in the odd gaps of elements of $\mathfrak{S}_{2 m}-\mathcal{A}_{2 m}$. This way, after insertion, " $n$ " will appear in an even position in $\mathcal{A}_{n}$.

Lemma 12. For a positive integer $m$, let $n=2 m$. Then, $\pi \in \mathcal{A}_{n}$ arises either by placing " $n$ " in the last position of elements of $\mathcal{A}_{n-1}$, or by placing " $n$ " in the first position of elements of $\mathfrak{S}_{n-1}-\mathcal{A}_{n-1}$ or by placing " $n$ " in the odd gaps of elements of $\mathcal{A}_{n-1}$. This way, after insertion, " $n$ " will appear in an even position of $\mathcal{A}_{n}$. Another way to get $\pi \in \mathcal{A}_{n}$ is by placing " $n$ " in the even gaps of elements of $\mathfrak{S}_{n-1}-\mathcal{A}_{n-1}$. This way, after insertion, " $n$ " will appear in an odd position in $\mathcal{A}_{n}$.

Our recurrence relation for the polynomials $A_{n}^{+}(s, t)$ and $A_{n}^{-}(s, t)$ depends on the parity of $n$ and so we bifurcate the remaining part into two cases.

\subsection{When $n=2 m+1$}

We begin with the following.

Lemma 13. For $0 \leqslant r \leqslant m-1$, let $S_{r}=\left[\left(\mathfrak{S}_{2 m}-\mathcal{A}_{2 m}\right) \rightarrow \mathcal{A}_{2 m+1}^{2 r+1}\right]$ and $T_{r}=\left[\mathcal{A}_{2 m} \rightarrow\right.$ $\left.\left(\mathfrak{S}_{2 m+1}-\mathcal{A}_{2 m+1}\right)^{2 m-2 r-1}\right]$. Then, the following is true:

$$
\sum_{\pi \in S_{r}} t^{\operatorname{des}(\pi)} s^{\operatorname{asc}(\pi)}=\sum_{\pi \in T_{r}} t^{\operatorname{des}(\pi)} s^{\operatorname{asc}(\pi)} .
$$


Proof. It is clear that if $\pi \in \mathcal{A}_{2 m+1}^{2 r+1}$ then $\pi^{\prime} \in \mathfrak{S}_{2 m}-\mathcal{A}_{2 m}$ and that if $\pi \in\left(\mathfrak{S}_{2 m+1}-\right.$ $\left.\mathcal{A}_{2 m+1}\right)^{2 r+1}$ then $\pi^{\prime} \in \mathcal{A}_{2 m}$. We give a bijection $f: S_{r} \mapsto T_{r}$ such that $t^{\operatorname{des}(\pi)} s^{\operatorname{asc}(\pi)}=$ $t^{\operatorname{des}(f(\pi))} s^{\operatorname{asc}(f(\pi))}$ for all $\pi \in S_{r}$. Define

$$
f\left(\pi_{1}, \pi_{2}, \ldots, \pi_{2 r+1}, 2 m+1, \pi_{2 r+2}, \ldots, \pi_{2 m}\right)=\pi_{2 r+2}, \ldots, \pi_{2 m}, 2 m+1, \pi_{1}, \pi_{2}, \ldots, \pi_{2 r+1} .
$$

Let $\pi \in \mathcal{A}_{2 m+1}^{2 r+1}$ and let $K=\pi_{1}, \pi_{2}, \ldots, \pi_{2 r+1}$ be $\pi$ restricted to the first $2 r+1$ elements. Let $L=\pi_{2 r+2}, \ldots, \pi_{2 m}$ be $\pi$ restricted to last $2 m-2 r-1$ elements. Even though $K$ and $L$ are permutations, we abuse notation for our next definition and treat them as sets by ignoring the order among their elements. Define invpair $(K, L)=\mid\{(x, y)$ : $x \in K, y \in L, x>y\} \mid$ and $\operatorname{invpair}(L, K)=|\{(x, y): x \in L, y \in K, x>y\}|$. Clearly, $\operatorname{invpair}(K, L)+\operatorname{invpair}(L, K)=|K| \times|L|$. If we treat $K$ and $L$ as subpermutations of $\pi$, we have $\pi=K, 2 m+1, L$ and $f(\pi)=L, 2 m+1, K$. Thus,

$$
\begin{aligned}
\operatorname{inv}(\pi) & =\operatorname{inv}(K)+\operatorname{inv}(L)+2 m-(2 r+1)+\operatorname{invpair}(K, L) \\
\operatorname{inv}(f(\pi)) & =\operatorname{inv}(K)+\operatorname{inv}(L)+2 r+1+\operatorname{invpair}(L, K) \\
\operatorname{inv}(\pi)-\operatorname{inv}(f(\pi)) & =\operatorname{invpair}(K, L)+2 m-2(2 r+1)-\operatorname{invpair}(L, K) \\
& \equiv 1(\bmod 2) .
\end{aligned}
$$

The last line follows as both $K$ and $L$ have odd cardinality. Hence the number of pairs of elements in the cross product is odd. Thus, $\pi$ and $f(\pi)$ have opposite signs. Hence, $\pi \in \mathcal{A}_{2 m+1}$ iff $f(\pi) \in \mathfrak{S}_{2 m+1}-\mathcal{A}_{2 m+1}$. Define $g: T_{r} \mapsto S_{r}$ by

$g\left(\pi_{1}, \ldots, \pi_{2 m-2 r-1}, 2 m+1, \pi_{2 m-2 r}, \ldots, \pi_{2 m}\right)=\pi_{2 m-2 r}, \ldots, \pi_{2 m}, 2 m+1, \pi_{1}, \ldots, \pi_{2 m-2 r-1}$.

Clearly, $f$ is a bijection with $f^{-1}=g$. Further, for $\pi \in S_{r}$, it is clear that $t^{\operatorname{des}(\pi)} s^{\operatorname{asc}(\pi)}=$ $t^{\operatorname{des}(f(\pi))} s^{\operatorname{asc}(f(\pi))}$, completing the proof.

For $0 \leqslant r \leqslant m-1$, define

$$
P_{r}^{2 m+1}(s, t)=\sum_{\pi \in S_{r}} t^{\operatorname{des}(\pi)} s^{\operatorname{asc}(\pi)} \text { and } Q_{r}^{2 m+1}(s, t)=\sum_{\pi \in T_{r}} t^{\operatorname{des}(\pi)} s^{\operatorname{asc}(\pi)} .
$$

Summing Lemma 13 when $r$ runs from 0 to $m-1$, we get the following.

Corollary 14. For any odd natural number $2 m+1$, the following equality holds:

$$
\sum_{r=0}^{m-1} P_{r}^{2 m+1}(s, t)=\sum_{r=0}^{m-1} Q_{r}^{2 m+1}(s, t) .
$$

With these results, we can now prove the following.

Theorem 15. Similar to the recurrence (5) for $A_{n}(s, t)$, we get the following two recurrences for $A_{2 m+1}^{+}(s, t)$ and $A_{2 m+1}^{-}(s, t)$ respectively:

$$
\begin{aligned}
& A_{2 m+1}^{+}(s, t)=(s+t) A_{2 m}^{+}(s, t)+s t D A_{2 m}^{+}(s, t), \\
& A_{2 m+1}^{-}(s, t)=(s+t) A_{2 m}^{-}(s, t)+s t D A_{2 m}^{-}(s, t) .
\end{aligned}
$$


Proof. We prove (13) first. Since (13) is identical to (5), we will use ideas from the sketch of the proof of Theorem 8. As shown in that proof, the right hand side of (13) equals $\sum_{\pi} t^{\operatorname{des}(\pi)} s^{\operatorname{asc}(\pi)}$ where the summation is over $\pi$ with $\pi^{\prime} \in \mathcal{A}_{2 m}$.

Recall $A_{2 m+1}^{+}(s, t)=\sum_{\pi \in \mathcal{A}_{2 m+1}} t^{\operatorname{des}(\pi)} s^{\mathrm{asc}(\pi)}$. We consider the contribution to $A_{2 m+1}^{+}(s, t)$ from $\pi \in \mathcal{A}_{2 m+1}$ with the letter $2 m+1$ occurring in position $\ell$ for all possible choices of $\ell$.

By Lemma 11, the contribution from $\pi$ where $2 m+1$ appears in the first or the last position is accounted for by the terms $s A_{2 m}^{+}(s, t)$ and $t A_{2 m}^{+}(s, t)$ respectively. The remaining $\pi \in \mathcal{A}_{2 m+1}$ arise when either $2 m+1$ appears in even positions of $\mathcal{A}_{2 m}$ or when $2 m+1$ appears in odd positions of $\mathfrak{S}_{2 m}-\mathcal{A}_{2 m}$. The contribution of these two possibilities are accounted for by the two terms $\sum_{r=1}^{m-1}\left(\sum_{\pi \in \mathcal{A}_{2 m} \rightarrow \mathcal{A}_{2 m+1}^{2 r}} t^{\operatorname{des}(\pi)} s^{\operatorname{asc}(\pi)}\right)$ and $\sum_{r=0}^{m-1}\left(\sum_{\pi \in\left(\mathfrak{S}_{2 m}-\mathcal{A}_{2 m}\right) \rightarrow \mathcal{A}_{2 m+1}^{2 r+1}} t^{\operatorname{des}(\pi)} s^{\operatorname{asc}(\pi)}\right)$ respectively.

By $(12), \sum_{r=0}^{m-1}\left(\sum_{\pi \in\left(\mathfrak{S}_{2 m}-\mathcal{A}_{2 m}\right) \rightarrow \mathcal{A}_{2 m+1}^{2 r+1}} t^{\operatorname{des}(\pi)} s^{\operatorname{asc}(\pi)}\right)=\sum_{r=0}^{m-1} P_{r}^{2 m+1}(s, t)$. By Corollary 14 , this equals $\sum_{r=0}^{m-1} Q_{r}^{2 m+1}(s, t)$.

The sum of the terms $\sum_{r=1}^{m-1}\left(\sum_{\pi \in \mathcal{A}_{2 m} \rightarrow \mathcal{A}_{2 m+1}^{2 r}} t^{\operatorname{des}(\pi)} s^{\operatorname{asc}(\pi)}\right)$ and $\sum_{r=0}^{m-1} Q_{r}^{2 m+1}(s, t)$ clearly equals the sum over all $\pi$ with $2 m+1$ coming in all the positions of $\mathcal{A}_{2 m}$, completing the proof. An identical proof shows (14).

\subsection{When $n=2 m$}

The strategy and hence our moves are similar to the case when $n=2 m+1$. We will need to make minor changes due to the difference between Lemmas 11 and 12. We will sketch the strategy and omit some proofs that contain similar arguments. We start with the following counterpart of Lemma 13.

Lemma 16. For $0 \leqslant r \leqslant m-1$, let $K_{r}=\left[\mathcal{A}_{2 m-1} \rightarrow \mathcal{A}_{2 m}^{2 r+1}\right]$ and $L_{r}=\left[\mathcal{A}_{2 m-1} \rightarrow\right.$ $\left.\left(\mathfrak{S}_{2 m}-\mathcal{A}_{2 m}\right)^{2 m-2 r-2}\right]$. Let $U_{r}=\left[\left(\mathfrak{S}_{2 m-1}-\mathcal{A}_{2 m-1}\right) \rightarrow\left(\mathfrak{S}_{2 m-1}-\mathcal{A}_{2 m-1}\right)^{2 r+1}\right]$ and $V_{r}=$ $\left[\left(\mathfrak{S}_{2 m-1}-\mathcal{A}_{2 m-1}\right) \rightarrow \mathcal{A}_{2 m-1}^{2 m-2 r-2}\right]$. Then, we assert the following:

$$
\begin{aligned}
& \sum_{\pi \in K_{r}} t^{\operatorname{des}(\pi)} s^{\operatorname{asc}(\pi)}=\sum_{\pi \in L_{r}} t^{\operatorname{des}(\pi)} s^{\operatorname{asc}(\pi)} \\
& \sum_{\pi \in U_{r}} t^{\operatorname{des}(\pi)} s^{\operatorname{asc}(\pi)}=\sum_{\pi \in V_{r}} t^{\operatorname{des}(\pi)} s^{\operatorname{asc}(\pi)} .
\end{aligned}
$$

Proof. Define a bijection $f: K_{r} \mapsto L_{r}$ as follows:

$f\left(\pi_{1}, \pi_{2}, \ldots, \pi_{2 r+1}, 2 m, \pi_{2 r+2}, \ldots, \pi_{2 m-1}\right)=\pi_{2 r+2}, \ldots, \pi_{2 m-1}, 2 m, \pi_{1}, \pi_{2}, \ldots, \pi_{2 r+1}$. With this, the proof is identical to that of Lemma 13. We omit the details.

For $n=2 m$ and $0 \leqslant r \leqslant m-2$, define

$$
G_{r}^{2 m}(s, t)=\sum_{\pi \in K_{r}} t^{\operatorname{des}(\pi)} s^{\operatorname{asc}(\pi)}, \quad H_{r}^{2 m}(s, t)=\sum_{\pi \in L_{r}} t^{\operatorname{des}(\pi)} s^{\operatorname{asc}(\pi)}
$$




$$
I_{r}^{2 m}(s, t)=\sum_{\pi \in U_{r}} t^{\operatorname{des}(\pi)} s^{\operatorname{asc}(\pi)}, \quad J_{r}^{2 m}(s, t)=\sum_{\pi \in V_{r}} t^{\operatorname{des}(\pi)} s^{\operatorname{asc}(\pi)} .
$$

Summing over Lemma 16 when $0 \leqslant r \leqslant m-2$ gives the following corollary.

Corollary 17. For any even natural number $2 m$, the following two equalities hold:

$$
\sum_{r=0}^{m-2} G_{r}^{2 m}(s, t)=\sum_{r=0}^{m-2} H_{r}^{2 m}(s, t) \quad \text { and } \quad \sum_{r=0}^{m-2} I_{r}^{2 m}(s, t)=\sum_{r=0}^{m-2} J_{r}^{2 m}(s, t) .
$$

Theorem 18. Like recurrence (5) for $A_{n}(s, t)$, we get the following recurrences for $A_{2 m}^{+}(s, t)$ and $A_{2 m}^{-}(s, t)$ :

$$
\begin{aligned}
& A_{2 m}^{+}(s, t)=s A_{2 m-1}^{+}(s, t)+t A_{2 m-1}^{-}(s, t)+\frac{1}{2} s t D A_{2 m-1}(s, t) \\
& A_{2 m}^{-}(s, t)=s A_{2 m-1}^{-}(s, t)+t A_{2 m-1}^{+}(s, t)+\frac{1}{2} s t D A_{2 m-1}(s, t) .
\end{aligned}
$$

Proof. We prove (19) first. Recall $A_{2 m}^{+}(s, t)=\sum_{\pi \in \mathcal{A}_{2 m}} t^{\operatorname{des}(\pi)} s^{\operatorname{asc}(\pi)}$. We use Lemma 12 and consider the contribution to the right hand side of (19) from $\pi \in \mathcal{A}_{2 m}$ with the letter $2 m$ occurring in position $\ell$ for all possible choices of $\ell$.

Contribution from $\pi$ where $2 m$ appears in the first or the last position are accounted for by the terms $t A_{2 m-1}^{-}(s, t)$ and $s A_{2 m-1}^{+}(s, t)$ respectively. The remaining $\pi \in \mathcal{A}_{2 m}$ arise when $2 m$ appears in even positions of $\mathcal{A}_{2 m-1}$ or when $2 m$ appears in odd positions of $\mathfrak{S}_{2 m-1}-\mathcal{A}_{2 m-1}$. By Corollary 17, such permutations contribute

$$
\frac{1}{2} \sum_{r=0}^{m-2}\left(G_{r}^{2 m}(s, t)+H_{r}^{2 m}(s, t)+I_{r}^{2 m}(s, t)+J_{r}^{2 m}(s, t)\right) .
$$

This sum clearly equals $\frac{1}{2} s t D A_{2 m-1}(s, t)$ completing the proof.

Tanimoto (see Corollaries 3.3 and 4.2 of [22]) gave the following recurrence for the numbers $a_{n, k}^{+}$and $a_{n, k}^{-}$. These follow from Theorems 15 and 18.

Corollary 19 (Tanimoto). Let $n=2 m+1$ be an odd positive integer. Then, for $0 \leqslant k<$ $n$, we have

$$
a_{n, k}^{+}=(n-k) a_{n-1, k-1}^{+}+(k+1) a_{n-1, k}^{+} \quad \text { and } \quad a_{n, k}^{-}=(n-k) a_{n-1, k-1}^{-}+(k+1) a_{n-1, k}^{-} .
$$

Let $n=2 m$ be an even positive integer. Then, for $0 \leqslant k<n$, we have

$$
\begin{aligned}
& 2 a_{n, k}^{+}=(n-k+1) a_{n-1, k-1}^{-}+k a_{n-1, k}^{-}+(n-k-1) a_{n-1, k-1}^{+}+(k+2) a_{n-1, k}^{+}, \\
& 2 a_{n, k}^{-}=(n-k+1) a_{n-1, k-1}^{+}+k a_{n-1, k}^{+}+(n-k-1) a_{n-1, k-1}^{-}+(k+2) a_{n-1, k}^{-} .
\end{aligned}
$$




\section{Gamma positivity of $A_{n}^{+}(s, t)$ when $n \equiv 0,1(\bmod 4)$}

We are now ready to prove the first of our main results.

Proof of Theorem 1 : By Lemma 10, the polynomials $A_{n}^{+}(s, t)$ and $A_{n}^{-}(s, t)$ are palindromic iff $n \equiv 0,1(\bmod 4)$. Our proof is by induction on $n$ with the base case being $n=4$. In this case, it is easy to see that $A_{4}^{+}(s, t)=s^{3}+5 s^{2} t+5 s t^{2}+t^{3}$ and that $A_{4}^{-}(s, t)=6 s^{2} t+6 s t^{2}$. Both these are clearly gamma positive.

When $n \equiv 1(\bmod 4)$, the recurrence in Theorem 15 is clearly identical to Foata and Schützenberger's recurrence in Theorem 8. Thus, the same proof of Theorem 8 shows that both $A_{n}^{+}(s, t)$ and $A_{n}^{-}(s, t)$ are $\gamma$-positive with the same center of symmetry. To go from $n=4 k+1$ to $n=4 k+4$, we use both Theorem 18 and Theorem 15 to express $A_{4 k+4}^{+}(s, t)$ as a combination of terms $A_{4 k+1}(s, t)$ and its variants. Recall $D$ is the operator $\frac{\partial}{\partial s}+\frac{\partial}{\partial t}$. The following is easy to see.

$$
\begin{aligned}
A_{4 k+4}^{+}(s, t)= & L_{1}(s, t) A_{4 k+1}^{+}(s, t)+L_{2}(s, t) A_{4 k+1}^{-}(s, t)+L_{3}(s, t) D A_{4 k+1}^{+}(s, t) \\
& +L_{4}(s, t) D A_{4 k+1}^{-}(s, t)+L_{5}(s, t) D^{2} A_{4 k+1}(s, t)+L_{6}(s, t) D^{3} A_{4 k+1}(s, t)
\end{aligned}
$$

where the following table lists $L_{i}(s, t)$ and its center of symmetry.

\begin{tabular}{l|c}
$f(s, t)$ & center of symmetry of $f(s, t))$ \\
\hline$L_{1}(s, t)=(s+t)^{3}+2 s t(s+t)$ & $3 / 2$ \\
\hline$L_{2}(s, t)=6 s t(s+t)$ & $3 / 2$ \\
\hline$L_{3}(s, t)=2(s t)^{2}+4 s t(s+t)^{2}$ & 2 \\
\hline$L_{4}(s, t)=3 s t(s+t)^{2}+6(s t)^{2}$ & 2 \\
\hline$L_{5}(s, t)=3(s t)^{2}(s+t)$ & $5 / 2$ \\
\hline$L_{6}(s, t)=1 / 2(s t)^{3}$ & 3
\end{tabular}

Using Corollary 6, we get that each of the six terms in (21) has center of symmetry $2 k+$ $3 / 2$. Since the terms all have the same center of symmetry, the polynomial $A_{4 k+4}^{+}(s, t)$ is $\gamma$ positive. An identical proof works for $A_{4 k+4}^{-}(s, t)$ and one can check that both polynomials have the same center of symmetry. The proof is complete.

Several interpretations for the numbers $\gamma_{n, i}$ as cardinalities of appropriate sets are known (see Athanasiadis [2, Theorem 2.1]). We end this section with the following natural question arising from Theorem 1.

Question 20. Let $n \equiv 0,1(\bmod 4)$. We have $A_{n}^{+}(s, t)=\sum_{i=0}^{\lfloor(n-1) / 2\rfloor} \gamma_{n, i}^{+}(s t)^{i}(s+t)^{n-1-2 i}$ and $A_{n}^{-}(s, t)=\sum_{i=0}^{\lfloor(n-1) / 2\rfloor} \gamma_{n, i}^{-}(s t)^{i}(s+t)^{n-1-2 i}$. Can we get a combinatorial interpretation for the gamma coefficients $\gamma_{n, i}^{+}$or $\gamma_{n, i}^{-}$?

\section{$5 \quad$ When $n \equiv 2(\bmod 4)$}

When $n \equiv 2(\bmod 4)$, the polynomials $A_{n}^{+}(t)$ and $A_{n}^{-}(t)$ are not palindromic and so cannot be expressed in the gamma basis. Nonetheless, we show that both the above 
polynomials can be written as a sum of two gamma positive polynomials with different centers of symmetry. If a polynomial $h(t)$ is not palindromic, then we need at least two gamma positive summands with different centers of symmetry which will add up to give $h(t)$. Thus, the minimum number of summands needed is at least two. Surprisingly, when $n \equiv 2(\bmod 4)$ we show that this minimum number of summands is sufficient. We note that this result only holds for the univariate polynomials $A_{n}^{+}(t)$ and $A_{n}^{-}(t)$ and not for the bivariate counterparts $A_{n}^{+}(s, t)$ and $A_{n}^{-}(s, t)$.

\subsection{Sum of gamma positive polynomials}

Proof of Theorem 2 : We prove the result for $A_{4 m+2}^{+}(t)$. An identical proof works for $A_{4 m+2}^{-}(t)$. As $n=4 m+2$, by Theorem 18 we have

$$
\begin{aligned}
A_{4 m+2}^{+}(s, t) & =s A_{4 m+1}^{+}(s, t)+t A_{4 m+1}^{-}(s, t)+\frac{1}{2} s t D A_{4 m+1}(s, t) \text { and hence } \\
A_{4 m+2}^{+}(t) & =A_{4 m+1}^{+}(t)+t A_{4 m+1}^{-}(t)+\left.\left(\frac{1}{2} s t D A_{4 m+1}(s, t)\right)\right|_{s=1} .
\end{aligned}
$$

Let $p(s, t)=\frac{1}{2} s t D A_{4 m+1}(s, t)$. By Corollary $6, p(s, t)$ is $\gamma$-positive with center of symmetry $2 m-1 / 2+1$. Further, by Corollary 9 , all coefficients of $A_{4 m+1}(s, t)$ except the coefficient of $(s t)^{0}(s+t)^{4 m}$ are even. As the exponent $4 m$ will appear in $D(s+t)^{4 m}$ that coefficient will also be even. Hence $p(s, t)$ and $p(t)=\left.p(s, t)\right|_{s=1}$ are $\gamma$-positive with integral coefficients in the gamma basis. Further, $p(t)$ has center of symmetry $2 m+1 / 2$.

It is simple to see that $p(t)$ has odd length and thus by Lemma 7 , it can be written as $p(t)=p_{1}(t)+p_{2}(t)$ with respective centers of symmetry $2 m$ and $2 m+1$. Thus, $(22)$ becomes

$$
\begin{aligned}
A_{4 m+2}^{+}(t) & =A_{4 m+1}^{+}(t)+t A_{4 m+1}^{-}(t)+p_{1}(t)+p_{2}(t) \\
& =w_{1}(t)+w_{2}(t)
\end{aligned}
$$

where $w_{1}(t)=A_{4 m+1}^{+}(t)+p_{1}(t)$ has center of symmetry $2 m$ and $w_{2}(t)=t A_{4 m+1}^{-}(t)+p_{2}(t)$ has center of symmetry $2 m+1$. The proof is complete.

We remark that when $n \equiv 2(\bmod 4)$, the bivariate Eulerian polynomials when summed over the alternating group are not gamma positive in the stronger bivariate sense. We postpone the example to the end of the next subsection to avoid repeating the data (see Remark 23).

\subsection{A curious identity}

When $n \equiv 2(\bmod 4)$, Theorem 2 states that both the polynomials $A_{n}^{+}(t)$ and $A_{n}^{-}(t)$ can be written as a sum of two gamma positive polynomials while Theorem 8 states that $A_{n}(t)$ is gamma positive. Below, we see how these four gamma-positive polynomials arising from $A_{n}^{+}(t)$ and $A_{n}^{-}(t)$ add up to give a single gamma positive polynomial for $A_{n}(t)$. We illustrate this with an example when $n=6$. It is easy to see that

$$
A_{6}(t)=1+57 t+302 t^{2}+302 t^{3}+57 t^{4}+t^{5} \text { and that }
$$




$$
\begin{array}{c|rl}
A_{6}^{+}(t) & =1+29 t+147 t^{2}+155 t^{3}+28 t^{4} & A_{6}^{-}(t)=28 t+155 t^{2}+147 t^{3}+29 t^{4}+t^{5} \\
f_{1}(t)=1+29 t+120 t^{2}+29 t^{3}+t^{4} & g_{1}(t)=t+29 t^{2}+120 t^{3}+29 t^{4}+t^{5} \\
f_{2}(t)=27 t^{2}+126 t^{3}+27 t^{4} & g_{2}(t)=27 t+126 t^{2}+27 t^{3}
\end{array}
$$

In the gamma basis, we have

\begin{tabular}{l|l|l|l}
$A_{6}(t)$ & $(1+t)^{5}$ & $52 t(1+t)^{3}$ & $136 t^{2}(1+t)$ \\
\hline$g_{1}(t)$ & $t(1+t)^{4}$ & $25 t^{2}(1+t)^{2}$ & $64 t^{3}$ \\
$g_{2}(t)$ & & $27 t(1+t)^{2}$ & $72 t^{2}$ \\
\hline$f_{1}(t)$ & $(1+t)^{4}$ & $25 t(1+t)^{2}$ & $64 t^{2}$ \\
$f_{2}(t)$ & & $27 t^{2}(1+t)^{2}$ & $72 t^{3}$
\end{tabular}

Remark 21. From our proof, it is not hard to see that $t f_{1}(t)=g_{1}(t)$ and $t g_{2}(t)=g_{1}(t)$.

If $A_{4 m+2}^{+}(t)=f_{1}(t)+f_{2}(t)$ then, each gamma coefficient of $A_{4 m+2}(t)$ is the sum of a gamma coefficient of $f_{1}(t)$ and a gamma coefficient of $f_{2}(t)$. By Remark 21, each gamma coefficient of $A_{4 m+2}(t)$ is also a sum of two gamma coefficients, one each of $g_{1}(t)$ and $g_{2}(t)$. We prove this below.

Let $\gamma_{n, i}$ denote the $i$-th gamma coefficient when $A_{n}(t)$ is expressed in the $\Gamma$ basis. Similarly, let $A_{4 m+2}^{+}(t)=f_{1}(t)+f_{2}(t)$ with $f_{1}(t)=\sum_{i=0}^{2 m} \gamma_{4 m+2, i}^{+, 1} t^{i}(1+t)^{4 m-2 i}$ and with $f_{2}(t)=\sum_{i=0}^{2 m+1} \gamma_{4 m+2, i}^{+, 2} t^{i}(1+t)^{4 m-2 i+2}$. When $n \equiv 0,1(\bmod 4)$, let $A_{n}^{+}(t)=$ $\sum_{i=0}^{\lfloor(n-1) / 2\rfloor} \gamma_{n, i}^{+} t^{i}(1+t)^{n-1-2 i}$ and let $A_{n}^{-}(t)=\sum_{i=0}^{\lfloor(n-1) / 2\rfloor} \gamma_{n, i}^{-} t^{i}(1+t)^{n-1-2 i}$.

Lemma 22. Let $n=4 m+2$ where $m$ is a positive integer. With the above notation,

$$
\gamma_{4 m+2, i-1}=\gamma_{4 m+2, i-1}^{+, 1}+\gamma_{4 m+2, i}^{+, 2} \text {. }
$$

Proof. Equation (24) in the proof of Theorem 2 gives $A_{4 m+2}^{+}(t)=f_{1}(t)+f_{2}(t)$. The proof of Theorem 2 also shows that

$$
\begin{aligned}
f_{1}(t)= & \sum_{i} \frac{1}{2} \gamma_{4 m+1, i} i t^{i}(1+t)^{4 m-2 i}+\sum_{i} \gamma_{4 m+1, i}(4 m-2 i) t^{i+1}(1+t)^{4 m-2 i-2} \\
& +A_{4 m+1}^{+}(t), \\
f_{2}(t)= & \sum_{i} \frac{1}{2} \gamma_{4 m+1, i} i t^{i+1}(1+t)^{4 m-2 i-2}+\sum_{i} \gamma_{4 m+1, i}(4 m-2 i) t^{i+2}(1+t)^{4 m-2 i-4} \\
& +t A_{4 m+1}^{-}(t) .
\end{aligned}
$$

Thus,

$$
\begin{aligned}
\gamma_{4 m+2, i}^{\mathcal{A}, 1} & =\frac{i}{2} \gamma_{4 m+1, i}+(4 m-2(i-1)) \gamma_{4 m+1, i-1}+\gamma_{4 m+1, i}^{\mathcal{A}} \\
\gamma_{4 m+2, i}^{\mathcal{A}, 2} & =\frac{i-1}{2} \gamma_{4 m+1, i-1}+(4 m-2(i-2)) \gamma_{4 m+1, i-2}+\gamma_{4 m+1, i-1}^{\mathfrak{S}-\mathcal{A}}
\end{aligned}
$$

Hence, $\gamma_{4 m+2, i-1}^{\mathcal{A}, 1}+\gamma_{4 m+2, i}^{\mathcal{A}, 2}=[4 m-2(i-2)] \gamma_{4 m+1, i-1}+i \gamma_{4 m+1, i}=\gamma_{4 m+2, i-1}$, completing the proof. 
Remark 23 . When $n \equiv 2(\bmod 4)$, the bivariate Eulerian polynomials $A_{n}^{+}(s, t)$ and $A_{n}^{-}(s, t)$ are not $\gamma$-positive. For example, when $n=6$, our proof gives

$$
\begin{aligned}
A_{6}^{+}(s, t) & =s^{5}+29 s^{4} t+147 s^{3} t^{2}+155 s^{2} t^{3}+28 s t^{4} \text { with } \\
f_{1}(s, t) & =s(s+t)^{4}+25 s^{2} t(s+t)^{2}+64 s^{3} t^{2} \text { and } \\
f_{2}(s, t) & =27 s t^{2}(s+t)^{2}+72 s^{2} t^{3} .
\end{aligned}
$$

Thus, though $A_{6}^{+}(t)=\left.A_{6}^{+}(s, t)\right|_{s=1}$ is a sum of two gamma positive polynomials, $A_{6}^{+}(s, t)$ is not.

\section{$6 \quad$ When $n \equiv 3(\bmod 4)$}

When $n \equiv 3(\bmod 4)$, we are unable to write $A_{n}(t)$ as a sum of two gamma positive polynomials, but can write it as a sum of three such polynomials. We start with the following example.

Example 24. We write polynomials in a tabular form for ease of reading. Let $n=7$. It can be checked that $A_{7}^{+}(t)$ is the polynomial given below and define the polynomials $f_{i}(t)$ as follows:

$$
\begin{aligned}
& A_{7}^{+}(t)=56 t^{5}+605 t^{4}+1208 t^{3}+586 t^{2}+64 t \quad+1, \\
& f_{1}(t)=54 t^{5}+432 t^{4}+54 t^{3}, \\
& f_{2}(t)=2 t^{5}+172 t^{4}+1092 t^{3}+172 t^{2}+2 t,
\end{aligned}
$$

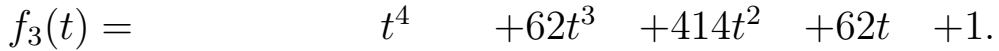

It can be readily checked that each $f_{i}(t)$ is $\gamma$-positive and that their sum equals $A_{7}^{+}(t)$.

Our main result of this section is the following counterpart of Theorem 2 which shows that three gamma positive summands are always sufficient.

Proof of Theorem 3 : By Theorem 15, $A_{4 m+3}^{+}(s, t)=(s+t) A_{4 m+2}^{+}(s, t)+s t D A_{4 m+2}^{+}(s, t)$. We handle the two terms separately. By Theorem 18,

$$
\begin{aligned}
& \left.(s+t) A_{4 m+2}^{+}(s, t)\right|_{s=1} \\
= & \left.\left\{(s+t)\left(s A_{4 m+1}^{+}(s, t)+t A_{4 m+1}^{-}(s, t)+\frac{s t}{2} D A_{4 m+1}(s, t)\right)\right\}\right|_{s=1} \\
= & (1+t)\left[A_{4 m+1}^{+}(t)+t A_{4 m+1}^{-}(t)\right]+\left.\left\{\frac{s t}{2}(s+t) D A_{4 m+1}(s, t)\right\}\right|_{s=1} .
\end{aligned}
$$

Let $p_{1}(t)=(1+t) A_{4 m+1}^{+}(t)$,

$$
p_{2}(t)=t(1+t) A_{4 m+1}^{-}(t) \text { and } p_{3}(t)=\left.\left\{\frac{s t}{2}(s+t) D A_{4 m+1}(s, t)\right\}\right|_{s=1} .
$$

By Corollary 6 , the three polynomials $p_{1}(t), p_{2}(t)$ and $p_{3}(t)$ have respective centers of symmetry $2 m+\frac{1}{2}, 2 m+\frac{3}{2}$ and $2 m+1$. Next, we consider the second term. 


$$
\begin{aligned}
\left.s t D A_{4 m+2}^{+}(s, t)\right|_{s=1}= & \left.s t D\left(s A_{4 m+1}^{+}(s, t)+t A_{4 m+1}^{-}(s, t)+\frac{s t}{2} D A_{4 m+1}(s, t)\right)\right|_{s=1} \\
= & \left(s^{2} t D A_{4 m+1}^{+}(s, t)+s t A_{4 m+1}^{+}(s, t)+s t^{2} D A_{4 m+1}^{-}(s, t)\right. \\
& +s t A_{4 m+1}^{-}(s, t)+\left.s t D\left(\frac{1}{2} s t D A_{4 m+1}(s, t)\right)\right|_{s=1} \\
= & \left.s^{2} t D A_{4 m+1}^{+}(s, t)\right|_{s=1}+t A_{4 m+1}^{+}(t)+\left.s t^{2} D A_{4 m+1}^{-}(s, t)\right|_{s=1} \\
& +t A_{4 m+1}^{-}(s, t)+\left.s t D\left(\frac{1}{2} s t D A_{4 m+1}(s, t)\right)\right|_{s=1} .
\end{aligned}
$$

Define

$$
\begin{aligned}
& p_{4 m+3}^{1}(t)=p_{1}(t)+\left.\left(s^{2} t D A_{4 m+1}^{+}(s, t)\right)\right|_{s=1}, \\
& p_{4 m+3}^{2}(t)=t A_{4 m+1}^{+}(t)+t A_{4 m+1}^{-}(t)+p_{3}(t)+\left.\left(s t D \frac{s t}{2} D A_{4 m+1}(s, t)\right)\right|_{s=1}, \\
& p_{4 m+3}^{3}(t)=p_{2}(t)+\left.\left(s t^{2} D A_{4 m+1}^{-}(s, t)\right)\right|_{s=1} .
\end{aligned}
$$

By Corollary $6, p_{4 m+3}^{1}(t), p_{4 m+3}^{2}(t)$ and $p_{4 m+3}^{3}(t)$ have respective centers of symmetry $2 m+\frac{1}{2}, 2 m+1$ and $2 m+\frac{3}{2}$. Clearly adding (28) and (29) is equivalent to adding (30), (31) and (32). Thus, $A_{4 m+3}^{+}(t)$ can be written as a sum of three $\gamma$-positive polynomials. A very similar proof can be given to show that $A_{4 m+3}^{-}(t)$ can be written as a sum of three $\gamma$-positive polynomials. The proof is complete.

\section{Type B Coxeter Groups}

Recall that $\mathfrak{B}_{n}$ is the set of permutations $\pi$ of $T_{n}=\{-n,-(n-1), \ldots,-1,1,2, \ldots n\}$ that satisfy $\pi(-i)=-\pi(i)$. $\mathfrak{B}_{n}$ is referred to as the hyperoctahedral group or the group of signed permutations on $[n]$. For $\pi \in \mathfrak{B}_{n}$, for $1 \leqslant i \leqslant n$, we alternatively denote $\pi(i)$ as $\pi_{i}$ and for $1 \leqslant k \leqslant n$, denote $-k$ as $\bar{k}$. For $\pi=\pi_{1}, \pi_{2}, \ldots, \pi_{n} \in \mathfrak{B}_{n}$, define $\operatorname{Negs}(\pi)=\left\{\pi_{i}: i>0, \pi_{i}<0\right\}$ as the set of elements which occur in $\pi$ with a negative sign.

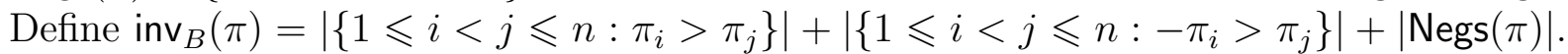
We refer to $\operatorname{inv}_{B}(\pi)$ alternatively as the length of $\pi \in \mathfrak{B}_{n}$. Let $\mathfrak{B}_{n}^{+} \subseteq \mathfrak{B}_{n}$ denote the subset of even length elements of $\mathfrak{B}_{n}$. Let $\mathfrak{B}_{n}^{-}=\mathfrak{B}_{n}-\mathfrak{B}_{n}^{+}$. For $\pi=\pi_{1}, \pi_{2}, \ldots, \pi_{n} \in \mathfrak{B}_{n}$, let $\pi_{0}=0$ and define its set of type $\mathrm{B}$ descents to be $\operatorname{DES}_{B}(\pi)=\left\{i \in\{0,1,2 \ldots, n-1\}: \pi_{i}>\pi_{i+1}\right\}$. Define the number of type B descents of $\pi$ to be $\operatorname{des}_{B}(\pi)=\left|\operatorname{DES}_{B}(\pi)\right|$ and the number of type B ascents of $\pi$ to be $\operatorname{asc}_{B}(\pi)=n-\operatorname{des}_{B}(\pi)$. Define

$$
B_{n}(t)=\sum_{\pi \in \mathfrak{B}_{n}} t^{\operatorname{des}_{B}(\pi)} \quad \text { and } \quad B_{n}(s, t)=\sum_{\pi \in \mathfrak{B}_{n}} t^{\operatorname{des}_{B}(\pi)} s^{\operatorname{asc}_{B}(\pi)}=\sum_{k=0}^{n} b_{n, k} t^{k} s^{n-k}
$$




$$
\begin{aligned}
& B_{n}^{+}(t)=\sum_{\pi \in \mathfrak{B}_{n}^{+}} t^{\operatorname{des}_{B}(\pi)} \quad \text { and } \quad B_{n}^{+}(s, t)=\sum_{\pi \in \mathfrak{B}_{n}^{+}} t^{\operatorname{des}_{B}(\pi)} s^{\operatorname{asc}_{B}(\pi)}=\sum_{k=0}^{n} b_{n, k}^{+} t^{k} s^{n-k} \\
& B_{n}^{-}(t)=\sum_{\pi \in \mathfrak{B}_{n}^{-}} t^{\operatorname{des}_{B}(\pi)} \quad \text { and } \quad B_{n}^{-}(s, t)=\sum_{\pi \in \mathfrak{B}_{n}^{-}} t^{\operatorname{des}_{B}(\pi)} s^{\operatorname{asc}_{B}(\pi)}=\sum_{k=0}^{n} b_{n, k}^{-} t^{k} s^{n-k} .
\end{aligned}
$$

The polynomial $B_{n}(t)$ is called the type B Eulerian polynomial. Gamma positivity of the type B Eulerian polynomial was shown by Chow [5] and Petersen [14]. Hyatt in [10] has given recurrences for the type $\mathrm{B}$ and type D Eulerian polynomials. We start with a type B counterpart of Foata and Schützenberger's recurrence (equation (5)) for the bivariate Eulerian polynomial. We could not find such a type B counterpart of equation (5) in the literature. It will be clear that (37) is such a type B counterpart. Towards that, we start with the following.

Theorem 25. With $B_{n}(s, t)$ as defined in (33), we have

$$
B_{n}(s, t)=\sum_{i=0}^{\lfloor n / 2\rfloor} \gamma_{n, i}^{B}(s t)^{i}(s+t)^{n-2 i}
$$

where the $\gamma_{n, i}^{B}$ are non negative integers for all $n, i$.

Proof. Recall $D=\frac{\partial}{\partial s}+\frac{\partial}{\partial t}$. We start by proving the following type B counterpart of (5).

$$
B_{n+1}(s, t)=(s+t) B_{n}(s, t)+2 s t D B_{n}(s, t) .
$$

Observe that the term $(s+t) B_{n}(s, t)$ is the contribution from $\pi \in \mathfrak{B}_{n}$ with either the letter ' $n+1$ ' or $\overline{n+1}$ at the last position. Further, it is not hard to see that $\operatorname{st} D B_{n}(s, t)$ is the contribution of all $\pi \in \mathfrak{B}_{n}$ in which the letter ' $n+1$ ' appears in position $1 \leqslant r \leqslant n$ and $\operatorname{st} D B_{n}(s, t)$ is the contribution of all $\pi \in \mathfrak{B}_{n}$ in which the letter $\overline{n+1}$ appears in position $r$ for $1 \leqslant r \leqslant n$. This completes the proof of (37).

It is straightforward to check that $B_{1}(s, t)=s+t$ and is thus $\gamma$-positive. Hence, by induction and Corollary 6 , we get that $B_{n+1}(s, t)$ is $\gamma$-positive. It is not hard to see that the following recurrence is satisfied by the coefficients $\gamma_{n+1, i}^{B}$.

$$
\gamma_{n+1, i}^{B}=(2 i+1) \gamma_{n, i}^{B}+4[n-2(i-1)] \gamma_{n, i-1}^{B} .
$$

The above recurrence together with the initial conditions $\gamma_{1,0}^{B}=1$ or $\gamma_{2,0}^{B}=1, \gamma_{2,1}^{B}=4$ settles non-negativity of $\gamma_{n, i}^{B}$ for all $n, i$.

Our next result showing palindromicity of the polynomials $B_{n}^{+}(s, t)$ and $B_{n}^{-}(s, t)$ is a type B counterpart of Lemma 10.

Lemma 26. When $n \geqslant 2$, the polynomials $B_{n}^{+}(s, t)$ and $B_{n}^{-}(s, t)$ are palindromic iff $n \equiv 0$ $\bmod 2$. 
Proof. Let $\pi=\pi_{1}, \pi_{2}, \ldots, \pi_{n} \in \mathfrak{B}_{n}$. Define $f: \mathfrak{B}_{n} \mapsto \mathfrak{B}_{n}$ by $f(\pi)=\overline{\pi_{1}}, \overline{\pi_{2}}, \ldots, \overline{\pi_{n}}$. Clearly, $f$ is a bijection. It is easy to see that flipping the sign of a single $\pi_{i}$ changes the parity of the number of type B inversions (for example, see [20, Lemma 3]). Further, it is easy to see that $\operatorname{des}_{B}(\pi)+\operatorname{des}_{B}(f(\pi))=n$. Hence, if $\pi \in \mathfrak{B}_{n}^{+}$then $f(\pi) \in \mathfrak{B}_{n}^{+}$iff $n \equiv 0$ $\bmod 2$.

If $n \equiv 1 \bmod 2$, then the map $f$ flips the parity of $\operatorname{inv}_{B}(\pi)$ and hence $b_{n, k}^{+}=b_{n, n-k}^{-}$. Further, $b_{n, 0}^{+}=1, b_{n, n}^{+}=0$ and $b_{n, n}^{-}=1, b_{n, 0}^{-}=0$ and hence $B_{n}^{+}(s, t)$ and $B_{n}^{-}(s, t)$ are not palindromic.

Define

$$
\operatorname{SgnB}_{n}(s, t)=\sum_{\pi \in \mathfrak{B}_{n}}(-1)^{\operatorname{inv}_{B}(\pi)} t^{\operatorname{des}_{B}(\pi)} s^{\operatorname{asc}_{B}(\pi)} .
$$

Clearly, $B_{n}^{+}(s, t)=\frac{1}{2}\left(B_{n}(s, t)+\operatorname{SgnB}_{n}(s, t)\right)$. Motivated by this, we determine $\operatorname{SgnB}_{n}(s, t)$ in the next subsection.

\subsection{Enumeration of descents with sign in $\mathfrak{B}_{n}$}

Let $a_{1}, a_{2}, \ldots, a_{n}$ be $n$ distinct positive integers with $a_{1}<a_{2}<\cdots<a_{n}$. Let $\mathfrak{B}_{\left\{a_{1}, a_{2}, \ldots, a_{n}\right\}}$ be the Type-B group of $2^{n} n$ ! permutations on the letters $a_{1}, a_{2}, \ldots, a_{n}$. Clearly, when $a_{i}=i$ for all $i$, we get $\mathfrak{B}_{n}=\mathfrak{B}_{\{1,2, \ldots, n\}}$. We write $\pi \in \mathfrak{B}_{\left\{a_{1}, a_{2}, \ldots, a_{n}\right\}}$ in two line notation with $a_{1}, a_{2}, \ldots, a_{n}$ above and $\pi_{a_{i}}$ below $a_{i}$. Thus, $\pi_{a_{i}}$ is defined for all $i$. We write

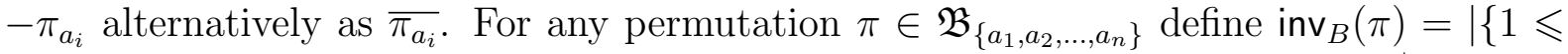
$\left.\left.i<j \leqslant n: \pi_{a_{i}}>\pi_{a_{j}}\right\}|+|\left\{1 \leqslant i<j \leqslant n:-\pi_{a_{i}}>\pi_{a_{j}}\right\} \mid\right\}+|\operatorname{Negs}(\pi)|$. Let $\mathfrak{B}_{\left\{a_{1}, a_{2}, \ldots, a_{n}\right\}}^{+}$ denote the subset of even length elements of $\mathfrak{B}_{\left\{a_{1}, a_{2}, \ldots, a_{n}\right\}}$ and $\mathfrak{B}_{\left\{a_{1}, a_{2}, \ldots, a_{n}\right\}}^{-}=\mathfrak{B}_{\left\{a_{1}, a_{2}, \ldots, a_{n}\right\}}-$ $\mathfrak{B}_{\left\{a_{1}, a_{2}, \ldots, a_{n}\right\}}^{+}$. Let $a_{0}=0$ and $\pi(0)=0$ for all $\pi$. Define $\operatorname{DES}_{B}(\pi)=\{i \in\{0,1,2 \ldots, n-1\}$ : $\left.\pi_{a_{i}}>\pi_{a_{i+1}}\right\}$ and $\operatorname{des}_{B}(\pi)=\left|D_{B E S}(\pi)\right|$. Let $\operatorname{asc}_{B}(\pi)=\mid\left\{i \in\{0,1,2 \ldots, n-1\}: \pi_{a_{i}}<\right.$ $\left.\pi_{a_{i+1}}\right\} \mid$. Let $\operatorname{pos}_{a_{i}}(\pi)=k$ if $\left|\pi_{a_{k}}\right|=a_{i}$ and define

$$
\operatorname{SgnB}_{\left\{a_{1}, a_{2}, \ldots, a_{n}\right\}}(s, t, u)=\sum_{\pi \in \mathfrak{B}_{\left\{a_{1}, a_{2}, \ldots, a_{n}\right\}}}(-1)^{\operatorname{inv}_{B}(\pi)} t^{\operatorname{des}_{B}(\pi)} s^{\operatorname{asc}_{B}(\pi)} u^{\operatorname{pos}_{a_{n}}(\pi)} .
$$

For positive integers $a_{1}<a_{2}<\ldots<a_{n}$, let $S=\left\{\pi \in \mathfrak{B}_{\left\{a_{1}, a_{2}, \ldots, a_{n}\right\}}^{+}, \operatorname{pos}_{a_{n}}(\pi) \neq n\right\}$ and $T=\left\{\pi \in \mathfrak{B}_{\left\{a_{1}, a_{2}, \ldots, a_{n}\right\}}^{-}, \operatorname{pos}_{a_{n}}(\pi) \neq n\right\}$. To evaluate (40), we will show that permutations with $a_{n}$ not at the last position do not contribute anything to $\operatorname{SgnB}_{\left\{a_{1}, a_{2}, \ldots, a_{n}\right\}}(s, t, u)$. This is done in Lemma 28 by partitioning $S$ and $T$ into three sets each and giving bijections that preserve $\mathrm{DES}_{B}$ and $\operatorname{pos}_{a_{n}}(\pi)$ between appropriate sets. One of the parts of $S$ and $T$ will be as follows. Let

$$
\begin{gathered}
S^{1}=\mathfrak{B}_{\left\{a_{1}, a_{2}, \ldots, a_{n}\right\}, \operatorname{pos}_{a_{n-1}}(\pi)=n, \operatorname{pos}_{a_{n}}(\pi)=n-1}^{+} \text {and } \\
T^{1}=\mathfrak{B}_{\left\{a_{1}, a_{2}, \ldots, a_{n}\right\}, \operatorname{pos}_{a_{n-1}}(\pi)=n, \operatorname{pos}_{a_{n}}(\pi)=n-1}^{-} .
\end{gathered}
$$

Clearly $S^{1} \subseteq S$ and $T^{1} \subseteq T$ 
Lemma 27. Let $n \geqslant 2$. Then, there exists a bijection $h_{1}: S^{1} \mapsto T^{1}$ such that for all $\pi \in S^{1}$, we have $\operatorname{DES}_{B}(\pi)=\operatorname{DES}_{B}\left(h_{1}(\pi)\right)$, $\operatorname{pos}_{a_{n}}(\pi)=\operatorname{pos}_{a_{n}}\left(h_{1}(\pi)\right)$ and $\operatorname{inv}_{B}(\pi) \not \equiv$ $\operatorname{inv}_{B}\left(h_{1}(\pi)\right)(\bmod 2)$. This will show

$$
\sum_{\pi \in S^{1} \cup T^{1}}(-1)^{\operatorname{inv}_{B}(\pi)} t^{\operatorname{des}_{B}(\pi)} s^{\operatorname{asc}_{B}(\pi)} u^{\operatorname{pos}_{a_{n}}(\pi)}=0 .
$$

Proof. Define $h_{1}: S^{1} \mapsto T^{1}$ by $h_{1}\left(\pi_{a_{1}}, \pi_{a_{2}}, \ldots, \pi_{a_{n-1}}, \pi_{a_{n}}\right)=\pi_{a_{1}}, \pi_{a_{2}}, \ldots, \pi_{a_{n-1}}, \overline{\pi_{a_{n}}}$. It is clear that $h_{1}$ is a bijection that preserves $\mathrm{DES}_{B}, \operatorname{pos}_{a_{n}}$ and flips the parity of inv $v_{B}$, completing the proof.

Our next lemma shows that $S \cup T$ does not contribute anything to the signed descent enumerator.

Lemma 28. There exists a bijection $h: S \mapsto T$ such that for all $\pi \in S$, we have $\operatorname{DES}_{B}(\pi)=\operatorname{DES}_{B}(h(\pi)), \operatorname{pos}_{a_{n}}(\pi)=\operatorname{pos}_{a_{n}}(h(\pi))$ and $\operatorname{inv}_{B}(\pi) \not \equiv \operatorname{inv}_{B}(h(\pi))(\bmod 2)$. This will show

$$
\sum_{\pi \in S \cup T}(-1)^{\operatorname{inv}_{B}(\pi)} t^{\operatorname{des}_{B}(\pi)} s^{\operatorname{asc}_{B}(\pi)} u^{\operatorname{pos}_{a_{n}}(\pi)}=0 .
$$

Proof. We use induction on $n$. When $n=2$, the required bijections are easy to construct. Let $n \geqslant 3$. For any set of $n-1$ distinct integers, by induction, assume that such a bijection exists. We partition $S$ as the disjoint union of the following three sets:

$$
\begin{gathered}
S^{1}=\left\{\pi \in \mathfrak{B}_{\left\{a_{1}, a_{2}, \ldots, a_{n}\right\}}^{+}, \operatorname{pos}_{a_{n-1}}(\pi)=n, \operatorname{pos}_{a_{n}}(\pi)=n-1\right\}, \\
S^{2}=\left\{\pi \in \mathfrak{B}_{\left\{a_{1}, a_{2}, \ldots, a_{n}\right\}}^{+}, \operatorname{pos}_{a_{n-1}}(\pi)=n, \operatorname{pos}_{a_{n}}(\pi) \neq n-1\right\} \text { and } \\
S^{3}=\left\{\pi \in \mathfrak{B}_{\left\{a_{1}, a_{2}, \ldots, a_{n}\right\}}^{+}, \operatorname{pos}_{a_{n-1}}(\pi) \neq n, \operatorname{pos}_{a_{n}}(\pi) \neq n\right\} .
\end{gathered}
$$

Similarly, we partition $T$ as the disjoint union of the following three sets:

$$
\begin{gathered}
T^{1}=\left\{\pi \in \mathfrak{B}_{\left\{a_{1}, a_{2}, \ldots, a_{n}\right\}}^{-}, \operatorname{pos}_{a_{n-1}}(\pi)=n, \operatorname{pos}_{a_{n}}(\pi)=n-1\right\}, \\
T^{2}=\left\{\pi \in \mathfrak{B}_{\left\{a_{1}, a_{2}, \ldots, a_{n}\right\}}^{-}, \operatorname{pos}_{a_{n-1}}(\pi)=n, \operatorname{pos}_{a_{n}}(\pi) \neq n-1\right\} \text { and } \\
T^{3}=\left\{\pi \in \mathfrak{B}_{\left\{a_{1}, a_{2}, \ldots, a_{n}\right\}}^{-}, \operatorname{pos}_{a_{n-1}}(\pi) \neq n, \operatorname{pos}_{a_{n}}(\pi) \neq n\right\} .
\end{gathered}
$$

Lemma 27, gives the required bijection $h_{1}: S^{1} \mapsto T^{1}$. We need the following two claims about bijections from $S^{2} \mapsto T^{2}$ and from $S^{3} \mapsto T^{3}$ respectively.

Claim 1: For all $n$ and positive integers $a_{1}<a_{2}<\cdots<a_{n}$, there exists a bijection $h_{2}: S^{2} \mapsto T^{2}$ satisfying $\operatorname{DES}_{B}(\pi)=\operatorname{DES}_{B}\left(h_{2}(\pi)\right), \operatorname{pos}_{a_{n}}(\pi)=\operatorname{pos}_{a_{n}}\left(h_{2}(\pi)\right)$ and $\operatorname{inv}_{B}(\pi) \not \equiv$ $\operatorname{inv}_{B}\left(h_{2}(\pi)\right)(\bmod 2)$.

Proof of Claim 1: By induction on the $n-1$ integers $a_{1}<a_{2}<\cdots<a_{n-2}<a_{n}$ there exists a bijection $g_{n-1}: \mathfrak{B}_{\left\{a_{1}, a_{2}, \ldots, a_{n-2}, a_{n}\right\}, \operatorname{pos}_{a_{n}}(\pi) \neq n-1}^{+} \rightarrow \mathfrak{B}_{\left\{a_{1}, a_{2}, \ldots, a_{n-2}, a_{n}\right\}, \operatorname{pos}_{a_{n}}(\pi) \neq n-1}$ 
with $\operatorname{DES}_{B}(\pi)=\operatorname{DES}_{B}\left(g_{n-1}(\pi)\right), \operatorname{pos}_{a_{n}}(\pi)=\operatorname{pos}_{a_{n}}\left(g_{n-1}(\pi)\right)$ and $\operatorname{inv}_{B}(\pi) \not \equiv \operatorname{inv}_{B}\left(g_{n-1}(\pi)\right)$ $(\bmod 2)$.

Define $h_{2}: S^{2} \mapsto T^{2}$ by $h_{2}\left(x_{1}, x_{2}, \ldots, x_{n-1}, x\right)=g_{n-1}\left(x_{1}, x_{2}, \ldots, x_{n-1}\right), x$ where $x=$ $a_{n-1}$ or $x=\overline{a_{n-1}}$. It is easy to check that $h_{2}$ is a bijection with the required properties. The proof of Claim 1 is complete.

Claim 2: For all $n$ and positive integers $a_{1}<a_{2}<\cdots<a_{n}$, there exists a bijection $h_{3}: S^{3} \mapsto T^{3}$ satisfying $\operatorname{DES}_{B}(\pi)=\operatorname{DES}_{B}\left(h_{3}(\pi)\right), \operatorname{pos}_{a_{n}}(\pi)=\operatorname{pos}_{a_{n}}\left(h_{3}(\pi)\right)$ and $\operatorname{inv}_{B}(\pi) \not \equiv$ $\operatorname{inv}_{B}\left(h_{3}(\pi)\right)(\bmod 2)$.

Proof of Claim 2: By induction on the $n-1$ integers, $a_{1}<a_{2}<\cdots<a_{n-2}<a_{n-1}$ there exists a bijection

$$
f_{n-1}: \mathfrak{B}_{\left\{a_{1}, a_{2}, \ldots, a_{n-2}, a_{n-1}\right\}, \operatorname{pos}_{a_{n-1}}(\pi) \neq n-1} \mapsto \mathfrak{B}_{\left\{a_{1}, a_{2}, \ldots, a_{n-2}, a_{n-1}\right\}, \operatorname{pos}_{a_{n-1}}(\pi) \neq n-1}
$$

with $\operatorname{DES}_{B}(\pi)=\operatorname{DES}_{B}\left(f_{n-1}(\pi)\right) \operatorname{inv}_{B}(\pi) \not \equiv \operatorname{inv}_{B}\left(f_{n-1}(\pi)\right)(\bmod 2)$ and $\operatorname{pos}_{a_{n-1}}(\pi)=$ $\operatorname{pos}_{a_{n-1}}\left(f_{n-1}(\pi)\right)$.

Let $\pi \in S^{3}$ with $\operatorname{pos}_{a_{n}}(\pi)=p$. Let $\pi^{\prime}$ be obtained from $\pi$ by deleting either $x=a_{n}$ or $x=\overline{a_{n}}$. Define $\sigma=h_{3}(\pi)$ by the following two step procedure: let $\sigma^{\prime}=f_{n-1}\left(\pi^{\prime}\right)$ and then insert $x$ at position $p$ in $\sigma^{\prime}$ to get $\sigma$. It is easy to check that $h_{3}: S^{3} \mapsto T^{3}$ is a bijection with the relevant properties. The proof of Claim 2 is complete.

Since $S^{1}, S^{2}$ and $S^{3}$ are disjoint, it is easy to put together the three bijections $h_{1}, h_{2}$ and $h_{3}$ given in Lemma 27, Claim 1 and Claim 2 respectively, to complete the proof.

In Lemma 28 , by setting $u=1$ and $a_{i}=i$ for $1 \leqslant i \leqslant n$ we get the following.

Corollary 29. For positive integers $n$, the following is true.

$$
\begin{aligned}
\sum_{\pi \in \mathfrak{B}_{n}^{+}, \operatorname{npos}(\pi) \neq n} t^{\operatorname{des}_{B}(\pi)} s^{\operatorname{asc}_{B}(\pi)} & =\sum_{\pi \in \mathfrak{B}_{n}^{-}, \operatorname{npos}(\pi) \neq n} t^{\operatorname{des}_{B}(\pi)} s^{\operatorname{asc}_{B}(\pi)} \\
& =\frac{1}{2} \sum_{\pi \in \mathfrak{B}_{n}, \mathrm{npos}(\pi) \neq n} t^{\operatorname{des}_{B}(\pi)} s^{\operatorname{asc}_{B}(\pi)} .
\end{aligned}
$$

\subsection{Main results for $\mathfrak{B}_{n}$}

Theorem 30. Let $n \geqslant 2$ be a positive integer. The polynomials $B_{n}^{+}(s, t)$ and $B_{n}^{-}(s, t)$ satisfy the following recurrence:

$$
\begin{aligned}
& B_{n}^{+}(s, t)=s B_{n-1}^{+}(s, t)+t B_{n-1}^{-}(s, t)+s t D B_{n-1}(s, t), \\
& B_{n}^{-}(s, t)=s B_{n-1}^{-}(s, t)+t B_{n-1}^{+}(s, t)+s t D B_{n-1}(s, t) .
\end{aligned}
$$

Proof. We prove (44) first. Recall $B_{n}^{+}(s, t)=\sum_{\pi \in \mathfrak{B}_{n}^{+}} t^{\operatorname{des}_{B}(\pi)} s^{\operatorname{asc}_{B}(\pi)}$. Consider the contribution to the right hand side from $\pi \in \mathfrak{B}_{n}^{+}$with ' $n$ ' or ' $\bar{n}$ ' occurring in position $k$ for all possible choices of $k$. It is easy to see that

$$
B_{n}^{+}(s, t)=s B_{n-1}^{+}(s, t)+t B_{n-1}^{-}(s, t)+\sum_{\pi \in \mathfrak{B}_{n}^{+}, \operatorname{npos}(\pi) \neq n} t^{\operatorname{des}_{B}(\pi)} s^{\operatorname{asc}_{B}(\pi)} .
$$


Here, $s B_{n-1}^{+}(s, t)$ accounts for the contribution of all $\pi \in \mathfrak{B}_{n}^{+}$in which the letter ' $n$ ' appears in the last position and $t B_{n-1}^{-}(s, t)$ is the contribution of all $\pi \in \mathfrak{B}_{n}^{+}$in which the letter ' $\bar{n}$ ' appears in the last position. Further, by Corollary 29 we get

$$
\begin{aligned}
B_{n}^{+}(s, t) & =s B_{n-1}^{+}(s, t)+t B_{n-1}^{-}(s, t)+\frac{1}{2} \sum_{\pi \in \mathfrak{B}_{n}, \operatorname{npos}(\pi) \neq n} t^{\operatorname{des}_{B}(\pi)} s^{\operatorname{asc}_{B}(\pi)} \\
& =s B_{n-1}^{+}(s, t)+t B_{n-1}^{-}(s, t)+s t D B_{n-1}(s, t) .
\end{aligned}
$$

The last line follows from the fact that $2 s t D B_{n-1}(s, t)$ is the contribution of all the permutations in $\mathfrak{B}_{n}$ where ' $n$ ' or ' $\bar{n}$ ' is not in the final position, completing the proof of (44). The proof of (45) is identical and hence omitted.

The following is the main result of this section.

Theorem 31. For positive integers $n \geqslant 2$, both $B_{n}^{+}(s, t)$ and $B_{n}^{-}(s, t)$ are $\gamma$-positive with the same center of symmetry $\frac{n}{2}$ iff $n \equiv 0(\bmod 2)$.

Proof. By Lemma 26, we need $n \equiv 0(\bmod 2)$ for palindromicity. We use induction on $n=2 m$ to show gamma positivity. The base case is when $n=2$. We have $B_{2}^{-}(s, t)=4 s t$ and $B_{2}^{+}(s, t)=s^{2}+2 s t+t^{2}$, both of which are $\gamma$-positive with center of symmetry 1 . By induction, assume that for $n-2=2 m-2$, both $B_{n-2}^{-}(s, t)$ and $B_{n-2}^{+}(s, t)$ are $\gamma$-positive with center of symmetry $m-1$. By applying the recurrence relations (44) and (45) twice, we get

$$
\begin{aligned}
B_{n}^{+}(s, t)= & \left(s^{2}+2 s t+t^{2}\right) B_{n-2}^{+}(s, t)+4 s t B_{n-2}^{-}(s, t) \\
& +4 s t(s+t) D B_{n-2}(s, t)+2 s^{2} t^{2} D^{2} B_{n-2}(s, t), \\
B_{n}^{-}(s, t)= & \left(s^{2}+2 s t+t^{2}\right) B_{n-2}^{-}(s, t)+4 s t B_{n-2}^{+}(s, t) \\
& +4 s t(s+t) D B_{n-2}(s, t)+2 s^{2} t^{2} D^{2} B_{n-2}(s, t) .
\end{aligned}
$$

Each of the four individual terms in (46) and (47) are gamma positive and have center of symmetry $m$. Thus, both $B_{2 m}^{+}(s, t)$ and $B_{2 m}^{-}(s, t)$ are $\gamma$-positive with center of symmetry $m$, completing the proof.

Theorem 32. For positive odd integers $n \geqslant 3, B_{n}^{-}(t)$ and $B_{n}^{+}(t)$ are the sum of two gamma positive polynomials.

Proof. $B_{n}^{+}(s, t)$ and $B_{n}^{-}(s, t)$ satisfy the recurrences (44) and (45) which are identical to the recurrences (19) and (20) which we used to prove Theorem 2. Hence, this proof follows in an identical manner to the proof of Theorem 2.

\subsubsection{Recurrences for signed descent numbers}

Similar to Tanimoto's recurrence (see Corollary 19), we can get a recurrence for the type $\mathrm{B}$ signed descent numbers $b_{n, k}^{+}$and $b_{n, k}^{-}$. We need a lemma before we prove the recurrence. Recalling $D=\frac{\partial}{\partial s}+\frac{\partial}{\partial t}$, we start with the following. 
Lemma 33. Let $n$ be a positive integer. We have $D B_{n}^{+}(s, t)=D B_{n}^{-}(s, t)$. Thus, we get $D B_{n}^{+}(s, t)=D B_{n}^{-}(s, t)=\frac{1}{2} D B_{n}(s, t)$.

Proof. We use induction on $n$. The result is easy when $n$ is at most 2. By induction, assume $D B_{n}^{+}(s, t)=D B_{n}^{-}(s, t)$. We will show the result for $n+1$.

$$
\begin{aligned}
D B_{n+1}^{+}(s, t) & =D\left(s B_{n}^{+}(s, t)+t B_{n}^{-}(s, t)+s t D B_{n}(s, t)\right) \\
& =s D B_{n}^{+}(s, t)+B_{n}^{+}(s, t)+t D B_{n}^{-}(s, t)+B_{n}^{-}(s, t)+D\left(s t D B_{n}(s, t)\right) \\
& =s D B_{n}^{-}(s, t)+B_{n}^{-}(s, t)+t D B_{n}^{+}(s, t)+B_{n}^{+}(s, t)+D\left(s t D B_{n}(s, t)\right) \\
& =D B_{n+1}^{-}(s, t) .
\end{aligned}
$$

In the third line above, we have used induction. The proof is complete.

Our next result is the following recurrence for the numbers $b_{n, k}^{+}$and $b_{n, k}^{-}$.

Lemma 34. For positive integers $n$ and $0 \leqslant k \leqslant n, b_{n, k}^{+}$and $b_{n, k}^{-}$satisfy the following recurrence relations:

$$
\begin{aligned}
& \text { 1. } b_{n, k}^{+}=2 k b_{n-1, k}^{-}+(2 n-2 k+1) b_{n-1, k-1}^{-}+b_{n-1, k}^{+} \text {, } \\
& \text { 2. } b_{n, k}^{-}=2 k b_{n-1, k}^{+}+(2 n-2 k+1) b_{n-1, k-1}^{+}+b_{n-1, k}^{-} .
\end{aligned}
$$

Proof. We only prove one of the recurrences. Let $\left[t^{k} s^{n-k}\right] f(s, t)$ denote the coefficient of $t^{k} s^{n-k}$ in the polynomial $f(s, t)$.

$$
\begin{aligned}
b_{n, k}^{+} & =\left[t^{k} s^{n-k}\right] B_{n}^{+}(s, t) \\
& =\left[t^{k} s^{n-k}\right]\left(s B_{n-1}^{+}(s, t)+t B_{n-1}^{-}(s, t)+s t D B_{n-1}(s, t)\right) \\
& =\left[t^{k} s^{n-k-1}\right] B_{n-1}^{+}(s, t)+\left[t^{k-1} s^{n-k}\right] B_{n-1}^{-}(s, t)+\left[t^{k-1} s^{n-k-1}\right] D B_{n-1}(s, t) \\
& =b_{n-1, k}^{+}+b_{n-1, k-1}^{-}+\left[t^{k-1} s^{n-k-1}\right]\left(2 D B_{n-1}^{-}(s, t)\right) \\
& =2 k b_{n-1, k}^{-}+(2 n-2 k+1) b_{n-1, k-1}^{-}+b_{n-1, k}^{+} .
\end{aligned}
$$

Here, the penultimate line follows from Lemma 33. The proof of the other part is identical and is hence omitted.

\section{Type D Coxeter groups}

Recall that $\mathfrak{D}_{n} \subseteq \mathfrak{B}_{n}$ is the subset of type B permutations that have an even number of negative signs. Let $w=w_{1}, w_{2}, \ldots, w_{n} \in \mathfrak{D}_{n}$. The following combinatorial definition of type D inversions is well known (see, for example, Petersen's book [15, Page 302]): $\operatorname{inv}_{D}(w)=\operatorname{inv}_{A}(w)+\left|\left\{1 \leqslant i<j \leqslant n:-w_{i}>w_{j}\right\}\right|$. Here $\operatorname{inv}_{A}(w)$ is computed with respect to the usual order on $\mathbb{Z}$. Let $\mathfrak{D}_{n}^{+}=\left\{w \in \mathfrak{D}_{n}\right.$ : $\operatorname{inv}_{D}(w)$ is even $\}$ and let $\mathfrak{D}_{n}^{-}=\mathfrak{D}_{n}-\mathfrak{D}_{n}^{+}$. Let $w \in \mathfrak{D}_{n}$. We can also think of $w$ as an element of $\mathfrak{B}_{n}$. Thus, even though $w \in \mathfrak{D}_{n}$, we have $\operatorname{inv}_{B}(w)$ as well. We will need the following alternate definition of $\operatorname{inv}_{B}(w)$ (see, for example, Petersen's book, [15, Page 294]): $\operatorname{inv}_{B}(w)=\operatorname{inv}_{A}(w)+\mid\{1 \leqslant i<j \leqslant n$ : 
$\left.-w_{i}>w_{j}\right\}|+| \operatorname{Negs}(w) \mid$. Since these definitions are combinatorial, we can apply them to elements $w \in \mathfrak{B}_{n}$ or to $w \in \mathfrak{D}_{n}$. Hence, we also have $\operatorname{inv}_{D}(w)$ when $w \in \mathfrak{B}_{n}$. From the above definition of $\operatorname{inv}_{B}(w)$ and $\operatorname{inv}_{D}(w)$ we get the following simple corollary which we will need later.

Corollary 35. Let $w \in \mathfrak{B}_{n}$. Then, $\operatorname{inv}_{B}(w)=\operatorname{inv}_{D}(w)+|\operatorname{Negs}(w)|$.

We next give the combinatorial definition of type $\mathrm{D}$ descents. Let $w=w_{1}, w_{2}, \ldots, w_{n} \in$ $\mathfrak{D}_{n}$. Define $\operatorname{des}_{D}(w)=\operatorname{des}(w)+\chi\left(w_{1}+w_{2}<0\right)$ where $\chi(Z)$ is a $0 / 1$ indicator function taking value 1 if $Z$ is true and taking value 0 otherwise. Here, $\operatorname{des}(w)$ is as defined in the type A case. See Petersen's book [15, Page 302] for this definition. For example, when $w=\overline{3} 5 \overline{1} 42$, then $\operatorname{inv}_{D}(w)=6$ and $\operatorname{des}_{D}(w)=2$. Let $\operatorname{asc}_{D}(w)=n-\operatorname{des}_{D}(w)$. Define

$$
\begin{aligned}
& D_{n}(t)=\sum_{w \in \mathfrak{D}_{n}} t^{\operatorname{des}_{D}(w)} \quad \text { and } \quad D_{n}(s, t)=\sum_{w \in \mathfrak{D}_{n}} t^{\operatorname{des}_{D}(w)} s^{\operatorname{asc}_{D}(w)}, \\
& D_{n}^{+}(t)=\sum_{w \in \mathfrak{D}_{n}^{+}} t^{\operatorname{des}_{D}(w)} \quad \text { and } \quad D_{n}^{+}(s, t)=\sum_{w \in \mathfrak{D}_{n}^{+}} t^{\operatorname{des}_{D}(w)} s^{\operatorname{asc}_{D}(w)}, \\
& D_{n}^{-}(t)=\sum_{w \in \mathfrak{D}_{n}^{-}} t^{\operatorname{des}_{D}(w)} \quad \text { and } \quad D_{n}^{-}(s, t)=\sum_{\pi \in \mathfrak{D}_{n}^{-}} t^{\operatorname{des}_{D}(\pi)} s^{\operatorname{asc}_{D}(\pi)} .
\end{aligned}
$$

The polynomials $D_{n}(t)$ are called the type D Eulerian polynomials. Stembridge [21] showed that the polynomials $D_{n}(t)$ are $\gamma$-positive when $n \geqslant 1$ (see Petersen [15, Section 13.4]). For $D_{n}(s, t)$ as defined (48), we are unable to get recurrences similar to (5) or (37). Our proof showing $\gamma$-positivity of $D_{n}^{+}(t)$ is inspired by the proof in Petersen's book [15, Section 13.4] that shows $\gamma$-positivity of $D_{n}(t)$.

We saw earlier that $w \in \mathfrak{D}_{n}$ has $\operatorname{inv}_{B}(w)$ as well. We consider a similar notion for $w \in \mathfrak{B}_{n}$ as well. If $w \in \mathfrak{B}_{n}$, then $w$ has both the type $\mathrm{B}$ and the type $\mathrm{D}$ inversion numbers (though the type $\mathrm{D}$ inversion number does not have Coxeter theoretic meaning). Define $\mathfrak{B}_{n, \mathfrak{D}}^{+}=\left\{w \in \mathfrak{B}_{n}: \operatorname{inv}_{D}(w)\right.$ is even $\}$ and $\mathfrak{B}_{n, \mathfrak{D}}^{-}=\left\{w \in \mathfrak{B}_{n}: \operatorname{inv}_{D}(w)\right.$ is odd $\}$.

Lemma 36. With the above notation, for positive integers $n$, we have

$$
\begin{aligned}
\sum_{w \in \mathfrak{D}_{n}^{+}} t^{\operatorname{des}_{D}(w)} s^{\operatorname{asc}_{D}(w)} & =\sum_{w \in\left(\mathfrak{B}_{n, \mathfrak{D}}^{+}-\mathfrak{D}_{n}^{+}\right)} t^{\operatorname{des}_{D}(w)} s^{\operatorname{asc}_{D}(w)}=\frac{1}{2} \sum_{w \in \mathfrak{B}_{n, \mathfrak{D}}^{+}} t^{\operatorname{des}_{D}(w)} s^{\operatorname{asc}_{D}(w)}, \\
\sum_{w \in \mathfrak{D}_{n}^{-}} t^{\operatorname{des}_{D}(w)} s^{\operatorname{asc}_{D}(w)} & =\sum_{w \in\left(\mathfrak{B}_{n, \mathfrak{D}}^{-}-\mathfrak{D}_{n}^{-}\right)} t^{\operatorname{des}_{D}(w)} s^{\operatorname{asc}_{D}(w)}=\frac{1}{2} \sum_{w \in \mathfrak{B}_{n, \mathfrak{D}}^{-}} t^{\operatorname{des}_{D}(w)} s^{\operatorname{asc}_{D}(w)} .
\end{aligned}
$$

Proof. We prove (51) first. For this, consider the bijection from $\mathfrak{D}_{n}^{+}$to $\mathfrak{B}_{n, \mathfrak{D}}^{+}-\mathfrak{D}_{n}^{+}$by flipping the sign of the first element. That is, consider the map $f: \mathfrak{D}_{n}^{+} \mapsto \mathfrak{B}_{n, \mathfrak{D}}^{+}-\mathfrak{D}_{n}^{+}$ given by $f\left(u_{1} u_{2} \ldots u_{n}\right)=\overline{u_{1}} u_{2} \ldots u_{n}$. Clearly $f^{2}=i d$. We note that :

$$
\begin{aligned}
\operatorname{inv}_{D}\left(\overline{u_{1}} u_{2} \ldots u_{n}\right) & =\operatorname{inv}_{B}\left(\overline{u_{1}} u_{2} \ldots u_{n}\right)-\left|\operatorname{Negs}\left(\overline{u_{1}} u_{2} \ldots u_{n}\right)\right| \\
& \equiv \operatorname{inv}_{B}\left(u_{1} u_{2} \ldots u_{n}\right)-\left|\operatorname{Negs}\left(u_{1} u_{2} \ldots u_{n}\right)\right|(\bmod 2)
\end{aligned}
$$




$$
\equiv \operatorname{inv}_{D}\left(u_{1} u_{2} \ldots u_{n}\right)(\bmod 2)
$$

The second step uses the fact that flipping the sign of a single $u_{i}$ changes the parity of the number of type B inversions (see [20, Lemma 3]). Moreover,

$$
\begin{aligned}
\operatorname{des}_{D}\left(\overline{u_{1}} u_{2} \ldots u_{n}\right) & =\operatorname{des}\left(\overline{u_{1}} u_{2} \ldots u_{n}\right)+\chi\left(\overline{u_{1}}+u_{2}<0\right) \\
& =\operatorname{des}\left(u_{1} u_{2} \ldots u_{n}\right)+\chi\left(u_{1}+u_{2}<0\right)=\operatorname{des}_{D}\left(u_{1} u_{2} \ldots u_{n}\right) .
\end{aligned}
$$

The last line above follows from a simple case based argument. Thus, by (53) and (54), the parity of $\operatorname{inv}_{D}$ and number of descents remain unchanged by $f$, completing the proof. The proof of (52) is similar and hence omitted.

Let $u \in \mathfrak{S}_{n}$ and let $\beta(u)$ denote the set of $2^{n}$ elements of $\mathfrak{B}_{n}$ obtained by adding negative signs to the entries of $u$ in all possible ways.

Lemma 37. Let $u \in \mathfrak{S}_{n}$. Then, for all $w \in \beta(u)$, either $w \in \mathfrak{B}_{n, \mathfrak{D}}^{+}$or $w \in \mathfrak{B}_{n, \mathfrak{D}}^{-}$. Further, for any $u \in \mathfrak{S}_{n}, w \in \mathfrak{B}_{n, \mathfrak{D}}^{+}$if and only if $u \in \mathcal{A}_{n}$.

Proof. The lemma is simple to check when $n \leqslant 2$. We thus assume $n \geqslant 3$. We need to show that all the $2^{n}$ elements of $\beta(u)$ have the same parity of the number of type $\mathrm{D}$ inversions. For any element $w=w_{1}, w_{2}, \ldots, w_{n} \in \beta(u)$, let $w_{\bar{l}}=w_{1}, w_{2}, \ldots, \overline{w_{l}}, \ldots, w_{n}$ denote the permutation obtained by flipping the sign of the $l$-th entry of $w$. We need to show for any $l$, that $w$ and $w_{\bar{l}}$ have the same parity of number of type $\mathrm{D}$ inversions. Let $w \in \beta(u)$. By Corollary 35, $\operatorname{inv}_{B}(w)=\operatorname{inv}_{D}(w)+|\operatorname{Negs}(w)|$. Similarly, $\operatorname{inv}_{B}\left(w_{\bar{l}}\right)=\operatorname{inv}_{D}\left(w_{\bar{l}}\right)+\left|\operatorname{Negs}\left(w_{\bar{l}}\right)\right|$. It is not hard to see that $\operatorname{inv}_{B}(w) \not \equiv \operatorname{inv}_{B}\left(w_{\bar{l}}\right)(\bmod 2)$ (for example, see [20, Lemma

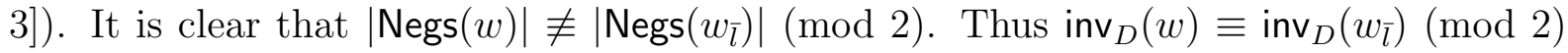
completing the proof.

By Lemma 37, we get

$$
\begin{gathered}
\sum_{w \in \mathfrak{B}_{n, \mathcal{D}}^{+}} t^{\operatorname{des}_{D}(w)} s^{\operatorname{asc}_{D}(w)}=\sum_{u \in \mathfrak{S}_{n} \cap \mathfrak{B}_{n, \mathfrak{D}}^{+}} \sum_{w \in \beta(u)} t^{\operatorname{des}_{D}(w)} s^{\operatorname{asc}_{D}(w)}=\sum_{u \in \mathcal{A}_{n}} \sum_{w \in \beta(u)} t^{\operatorname{des}_{D}(w)} s^{\operatorname{asc}_{D}(w)} \\
\sum_{w \in \mathfrak{B}_{n, \mathcal{D}}^{-}} t^{\operatorname{des}_{D}(w)} s^{\operatorname{asc}_{D}(w)}=\sum_{u \in \mathfrak{S}_{n} \cap \mathfrak{B}_{n, \mathcal{D}}^{-}} \sum_{w \in \beta(u)} t^{\operatorname{des}_{D}(w)}=\sum_{u \in \mathfrak{S}_{n}-\mathcal{A}_{n}} \sum_{w \in \beta(u)} t^{\operatorname{des}_{D}(w)} s^{\operatorname{asc}_{D}(w)} .
\end{gathered}
$$

The proof in Petersen's book [15, Page 305] of $\gamma$-positivity of $D_{n}(t)$ shows that it is possible to assign values $c_{i}(u)$ for $1 \leqslant i \leqslant n$ to $u \in \mathfrak{S}_{n}$ such that $\sum_{w \in \beta(u)} t^{\operatorname{des}_{D} w}=$ $c_{1}(u) c_{2}(u) \ldots c_{n}(u)$. We work with the following bivariate version.

$$
c_{1}(u) c_{2}(u)= \begin{cases}2 s^{2}+2 t^{2} & \text { if } u_{1}<u_{2}<u_{3} . \\ 2 s t(s+t) & \text { if } u_{1}<u_{2}>u_{3} . \\ 4 s t & \text { if } u_{1}>u_{2}<u_{3} . \\ 2 s t(s+t) & \text { if } u_{1}>u_{2}>u_{3} .\end{cases}
$$


and for all $j \geqslant 3$

$$
c_{j}(u)= \begin{cases}2 t & \text { if } u_{j-1}<u_{j}>u_{j+1} \\ 2 s & \text { if } u_{j-1}>u_{j}<u_{j+1} \\ s+t & \text { otherwise. }\end{cases}
$$

Recall that $n \geqslant 3$. For a permutation $u=u_{1}, u_{2}, u_{3}, \ldots, u_{n} \in \mathfrak{S}_{n}$ with $u_{1}<u_{2}<u_{3}$, let $u^{\prime}=u_{3}, u_{1}, u_{2}, \ldots, u_{n}$. It is easy to see that $u \in \mathfrak{B}_{n, \mathfrak{D}}^{+}$if and only if $u^{\prime} \in \mathfrak{B}_{n, \mathfrak{D}}^{+}$. We partition $\mathcal{A}_{n}$ into a union of the following five disjoint sets $\mathcal{A}_{n}^{1}=\left\{u=u_{1}, u_{2}, u_{3}, \ldots, u_{n} \in\right.$ $\left.\mathcal{A}_{n}: u_{1}<u_{2}>u_{3}\right\}, \mathcal{A}_{n}^{2}=\left\{u=u_{1}, u_{2}, u_{3}, \ldots, u_{n} \in \mathcal{A}_{n}: u_{1}>u_{2}>u_{3}\right\}, \mathcal{A}_{n}^{3}=\{u=$ $\left.u_{1}, u_{2}, u_{3}, \ldots, u_{n} \in \mathcal{A}_{n}: u_{1}<u_{2}<u_{3}\right\}, \mathcal{A}_{n}^{4}=\left\{u=u_{1}, u_{2}, u_{3}, \ldots, u_{n} \in \mathcal{A}_{n}: u_{1}>u_{2}<\right.$ $\left.u_{3}, u_{1}>u_{3}\right\}, \mathcal{A}_{n}^{5}=\left\{u=u_{1}, u_{2}, u_{3}, \ldots, u_{n} \in \mathcal{A}_{n}: u_{1}>u_{2}<u_{3}, u_{1}<u_{3}\right\}$. It is easy to see that $\mathcal{A}_{n}$ is indeed a disjoint union of these five sets. For a permutation $u \in \mathfrak{S}_{n}$, define its number of left peaks as $\operatorname{lpk}(u)=\left|\left\{1 \leqslant i<n: u_{i-1}<u_{i}>u_{i+1}\right\}\right|$ where $u_{0}=0$.

Lemma 38. For integers $n \geqslant 3,\left|\mathcal{A}_{n}^{3}\right|=\left|\mathcal{A}_{n}^{4}\right|$. Moreover, $\sum_{u \in \mathcal{A}_{n}^{3} \cup \mathcal{A}_{n}^{4}} \sum_{w \in \beta(u)} t^{\operatorname{des}_{D}(w)} s^{\operatorname{asc}_{D}(w)}$ is a $\gamma$-positive polynomial with all gamma coefficients being even.

Proof. Define $f: \mathcal{A}_{n}^{3} \mapsto \mathcal{A}_{n}^{4}$ by $f\left(u_{1}, u_{2}, u_{3}, u_{4}, \ldots, u_{n}\right)=u_{3}, u_{1}, u_{2}, u_{4}, \ldots, u_{n}$. Clearly, $\pi \in \mathcal{A}_{n}$ iff $f(\pi) \in \mathcal{A}_{n}$. Further, $f$ is a bijection as its inverse is clearly $g: \mathcal{A}_{n}^{4} \mapsto \mathcal{A}_{n}^{3}$ given by $g\left(u_{1}, u_{2}, u_{3}, u_{4}, \ldots, u_{n}\right)=u_{2}, u_{3}, u_{1}, u_{4}, \ldots, u_{n}$. Hence, we have

$$
\begin{aligned}
& \sum_{u \in \mathcal{A}_{n}^{3} \cup \mathcal{A}_{n}^{4}} \sum_{w \in \beta(u)} t^{\operatorname{des}_{D}(w)} s^{\operatorname{asc}_{D}(w)} \\
= & \sum_{u \in \mathcal{A}_{n}^{3}}\left(\sum_{w \in \beta(u)} t^{\operatorname{des}_{D}(w)} s^{\operatorname{asc}_{D}(w)}\right)+\sum_{f(u) \in \mathcal{A}_{n}^{4}}\left(\sum_{w \in \beta(f(u))} t^{\operatorname{des}_{D}(w)} s^{\operatorname{asc}_{D}(w)}\right) \\
= & \sum_{u \in \mathcal{A}_{n}^{3}}\left(2 s^{2}+2 t^{2}\right) \prod_{i=3}^{n} c_{i}(u)+\sum_{u \in \mathcal{A}_{n}^{3}} 4 s t \prod_{i=3}^{n} c_{i}(u)=\sum_{u \in \mathcal{A}_{n}^{3}} 2(s+t)^{2} \prod_{i=3}^{n} c_{i}(u) \\
= & \sum_{u \in \mathcal{A}_{n}^{3}} 2(4 s t)^{\operatorname{lpk}(u)}(s+t)^{n-2 \operatorname{lpk}(u)} .
\end{aligned}
$$

In the last line, we used bivariate version of [15, Eqn (13.10)] from Petersen's book. The proof is complete.

Theorem 39. For positive integers $n \geqslant 3$, the polynomials $D_{n}^{+}(s, t)$ and $D_{n}^{-}(s, t)$ are $\gamma$-positive.

Proof. We first prove that the polynomials $D_{n}^{+}(s, t)$ are $\gamma$-positive. From Lemma 36, we get

$$
\sum_{w \in \mathfrak{D}_{n}^{+}} t^{\operatorname{des}_{D}(w)} s^{\operatorname{asc}_{D}(w)}=\sum_{w \in\left(\mathfrak{B}_{n, \mathcal{D}}^{+}-\mathfrak{D}_{n}^{+}\right)} t^{\operatorname{des}_{D}(w)} s^{\operatorname{asc}_{D}(w)}=\frac{1}{2} \sum_{w \in \mathfrak{B}_{n, \mathcal{D}}^{+}} t^{\operatorname{des}_{D}(w)} s^{\operatorname{asc}_{D}(w)} .
$$


By (55), we get

$$
\begin{aligned}
\sum_{w \in \mathfrak{D}_{n}^{+}} t^{\operatorname{des}_{D}(w)} s^{\operatorname{asc}_{D}(w)}= & \frac{1}{2} \sum_{u \in \mathcal{A}_{n}}\left(\sum_{w \in \beta(u)} t^{\operatorname{des}_{D}(w)} s^{\operatorname{asc}_{D}(w)}\right) \\
= & \frac{1}{2} \sum_{i=1}^{5}\left(\sum_{u \in \mathcal{A}_{n}^{i}} \sum_{w \in \beta(u)} t^{\operatorname{des}_{D}(w)} s^{\operatorname{asc}_{D}(w)}\right) \\
= & \frac{1}{2} \sum_{i=1,2,5}\left(\sum_{u \in \mathcal{A}_{n}^{i}} \sum_{w \in \beta(u)} t^{\operatorname{des}_{D}(w)} s^{\operatorname{asc}_{D}(w)}\right) \\
& +\frac{1}{2} \sum_{i=3,4}\left(\sum_{u \in \mathcal{A}_{n}^{i}} \sum_{w \in \beta(u)} t^{\operatorname{des}_{D}(w)} s^{\operatorname{asc}_{D}(w)}\right) .
\end{aligned}
$$

As given in Petersen's book, [15, Eqn (13.9)] if $u_{1}<u_{2}>u_{3}$, or $u_{1}>u_{2}>u_{3}$ or $u_{3}>u_{1}>u_{2}$, then $\sum_{w \in \beta(u)} t^{\operatorname{des}_{D}(w)} s^{\operatorname{asc}_{D}(w)}=(4 s t)^{\operatorname{lpk}(u)}(s+t)^{n-2 \operatorname{lpk}(u)}$. So, the summation of $\operatorname{des}_{D}(w)$ in each of the three cases, that is, over $\mathcal{A}_{n}^{1}, \mathcal{A}_{n}^{2}$ and $\mathcal{A}_{n}^{5}$ is gamma positive and furthermore, $u$ in any of these three sets has at least one left peak. Thus, the gammacoefficients are even. Hence, the first term in (60) is gamma positive. Gamma positivity of the second term follows from Lemma 38. The proof for $\mathfrak{D}_{n}^{-}(s, t)$ follows identical steps and hence is omitted. The proof is complete.

\section{$9 \quad$ Unimodality of sequences}

A sequence $\left(a_{k}\right)_{k=0}^{n}$ is said to be unimodal if there exists an index $0 \leqslant r \leqslant n$ such that $a_{0} \leqslant a_{1} \leqslant \cdots \leqslant a_{r-1} \leqslant a_{r} \geqslant a_{r+1} \geqslant \cdots \geqslant a_{n}$. A polynomial is said to be unimodal if its sequence of coefficients is unimodal. In this short section, we prove that all polynomials defined in this paper are unimodal. We start with the following remark.

Remark 40. It is well known that $\gamma$-positivity of a polynomial implies unimodality of its coefficients (see Petersen's book [15, Chapter 4]).

Ma, Ma and Yeh in [12] show that if a polynomial $f(t)$ can be written as a sum of two gamma positive polynomials, then too the coefficients of $f(t)$ are unimodal. We need the centers of symmetry of the two summands to be separated by at most one for this. We show the following alternate argument.

Lemma 41. If $f(s, t), g(s, t)$ and $h(s, t)$ are $\gamma$-positive polynomials with the same center of symmetry, then, $s f(s, t)+t g(s, t)+\operatorname{stDh}(s, t)$ is unimodal.

Proof. We assume that the polynomials $f(s, t), g(s, t)$ and $h(s, t)$ have even degree as we will use this Lemma for showing $A_{4 m+2}^{+}(t)$ and $A_{4 m+2}^{-}(t)$ are unimodal. From the proof, it will be clear that there is no loss of generality in this assumption. Let

$$
f(s, t)=a_{2 m} t^{2 m}+a_{2 m-1} t^{2 m-1} s+\cdots+a_{m} t^{m} s^{m}+a_{m-1} t^{m-1} s^{m+1}+\cdots+a_{0} s^{2 m},
$$


with $a_{i} \leqslant a_{i+1}$ for $0 \leqslant i \leqslant m-1$ and $a_{i} \geqslant a_{i+1}$ for $m \leqslant i \leqslant 2 m-1$. Let

$$
g(s, t)=b_{2 m} t^{2 m}+b_{2 m-1} t^{2 m-1} s+\cdots+b_{m} t^{m} s^{m}+b_{m-1} t^{m-1} s^{m+1}+\cdots+b_{0} s^{2 m},
$$

where $b_{i} \leqslant b_{i+1}$ for $0 \leqslant i \leqslant m-1$ and $b_{i} \geqslant b_{i+1}$ for $m \leqslant i \leqslant 2 m-1$. By Lemma 5 Dh $(s, t)$ is a $\gamma$-positive whenever $h(s, t)$ is $\gamma$-positive. Let

$$
D h(s, t)=c_{2 m-1} t^{2 m-1}+c_{2 m-2} t^{2 m-2} s+\cdots+c_{m} t^{m} s^{m-1}+c_{m-1} t^{m-1} s^{m}+\cdots+c_{0} s^{2 m-1},
$$

with $c_{i} \leqslant c_{i+1}$ for $0 \leqslant i \leqslant m-1$ and $c_{i} \geqslant c_{i+1}$ for $m \leqslant i \leqslant 2 m-1$ and $c_{m-1}=c_{m}$. Hence, the coefficient of $t^{r} s^{2 m+1-r}$ in $s f(s, t)+t g(s, t)+s t D h(s, t)$ equals

$$
\begin{cases}b_{2 m} & \text { if } r=2 m+1 . \\ c_{r-1}+b_{r-1}+a_{r} & \text { if } 1 \leqslant r \leqslant 2 m \\ a_{0} & \text { if } r=0\end{cases}
$$

Clearly, the following two strings of inequality hold.

$$
\begin{gathered}
b_{2 m} \leqslant a_{2 m}+b_{2 m-1}+c_{2 m-1} \leqslant a_{2 m-1}+b_{2 m-2}+c_{2 m-2} \leqslant \cdots \leqslant a_{m+1}+b_{m}+c_{m} \text { and } \\
a_{m}+b_{m-1}+c_{m-1} \geqslant a_{m-1}+b_{m-2}+c_{m-2} \cdots \geqslant \cdots \geqslant a_{0} .
\end{gathered}
$$

Thus, $s f(s, t)+\operatorname{tg}(s, t)+\operatorname{st} D h(s, t)$ is unimodal.

Lemma 41 and Remark 40 give the following.

Corollary 42. The polynomials $A_{n}^{+}(t)$ and $A_{n}^{-}(t)$ are unimodal when $n \equiv 0,1,2$ (mod 4). When $n \geqslant 2$, the polynomials $B_{n}^{+}(t)$ and $B_{n}^{-}(t)$ are unimodal. When $n \geqslant 3$, the polynomials $D_{n}^{+}(t)$ and $D_{n}^{-}(t)$ are unimodal.

Proof. Combining Remark 40 with Theorem 1 completes the proof for unimodality of $A_{n}^{+}(t)$ and $A_{n}^{-}(t)$ when $n \equiv 0,1(\bmod 4)$. Combining Lemma 41 with Theorem 18 gives unimodality of $A_{n}^{+}(t)$ and $A_{n}^{-}(t)$ when $n \equiv 2(\bmod 4)$.

Using Theorem 31 and Theorem 32, we get unimodality of $B_{n}^{+}(t)$ and $B_{n}^{-}(t)$ when $n \geqslant 2$. Finally, Theorem 39 gives unimodality of $D_{n}^{+}(t)$ and $D_{n}^{-}(t)$ when $n \geqslant 3$.

Thus, unimodality of $A_{n}^{+}(t)$ and $A_{n}^{-}(t)$ when $n \equiv 3(\bmod 4)$ is the only case to be considered. We use Tanimoto's recurrence to show unimodality in this case.

Lemma 43. Let $a_{n, k}^{+}$and $a_{n, k}^{-}$denote the number of permutations in $\mathcal{A}_{n}$ and $\mathfrak{S}_{n}-\mathcal{A}_{n}$ with $k$ descents respectively. Then, we assert that:

1. if $a_{2 m, i-1}^{+} \leqslant a_{2 m, i}^{+}$and $a_{2 m, i}^{+} \leqslant a_{2 m, i+1}^{+}$, then $a_{2 m+1, i}^{+} \leqslant a_{2 m+1, i+1}^{+}$.

2. if $a_{2 m, i-1}^{+} \geqslant a_{2 m, i}^{+}$and $a_{2 m, i}^{+} \geqslant a_{2 m, i+1}^{+}$, then $a_{2 m+1, i}^{+} \geqslant a_{2 m+1, i+1}^{+}$.

3. if $a_{2 m, i-1}^{-} \leqslant a_{2 m, i}^{-}$and $a_{2 m, i}^{-} \leqslant a_{2 m, i+1}^{-}$, then $a_{2 m+1, i}^{-} \leqslant a_{2 m+1, i+1}^{-}$. 
4. if $a_{2 m, i-1}^{-} \geqslant a_{2 m, i}^{-}$and $a_{2 m, i}^{-} \geqslant a_{2 m, i+1}^{-}$, then $a_{2 m+1, i}^{-} \geqslant a_{2 m+1, i+1}^{-}$.

Thus, if $A_{4 m+2}^{+}(s, t)$ and $A_{4 m+2}^{-}(s, t)$ are unimodal, then so are $A_{4 m+3}^{+}(s, t)$ and $A_{4 m+3}^{-}(s, t)$.

Proof. We start by showing the first of the above assertions. Suppose, $a_{2 m, i-1}^{+} \leqslant a_{2 m, i}^{+}$and $a_{2 m, i}^{+} \leqslant a_{2 m, i+1}^{+}$. Using the recurrences proved by Tanimoto (see Corollary 19), we have

$$
\begin{aligned}
a_{2 m+1, i}^{+} & =(2 m+1-i) a_{2 m, i-1}^{+}+(i+1) a_{2 m, i}^{+} \\
& =(2 m+1-i-1) a_{2 m, i-1}^{+}+a_{2 m, i-1}^{+}+(i+2) a_{2 m, i}^{+}-a_{2 m, i}^{+} \\
& \leqslant a_{2 m+1, i+1}^{+}+a_{2 m, i-1}^{+}-a_{2 m, i}^{+} \leqslant a_{2 m+1, i+1}^{+} .
\end{aligned}
$$

Thus, the first statement is proved. In an identical manner, the other assertions can be proved.

The following is the main result of this section. One can check by hand that $D_{n}^{+}(t)$ is not unimodal when $n=2$.

Theorem 44. For positive integers $n$, the polynomials $A_{n}^{+}(s, t), A_{n}^{-}(s, t), B_{n}^{+}(s, t)$ and $B_{n}^{-}(s, t)$ are unimodal. When $n \geqslant 3$, the polynomials $D_{n}^{+}(s, t)$ and $D_{n}^{-}(s, t)$ are unimodal.

Proof. Corollary 42 and Lemma 43 take care of almost all cases. One can check by hand that $B_{n}^{+}(t)$ and $B_{n}^{-}(t)$ are unimodal when $n=1$. This completes the proof.

\section{Questions and Conjectures}

In this section, we raise a few questions and make a few conjectures. The most important question is to find an interpretation for gamma coefficients.

Question 45. Can we find a combinatorial interpretation for the gamma coefficients that occur in Theorems 2, 3, 31, 32 and 39 ?

Another question concerns $A_{4 m+3}^{+}(t)$. What is the minimum number of gamma positive summands needed to give $A_{4 m+3}^{+}(t)$ ? Theorem 3 shows that three such summands suffice, but are three needed?

Question 46. Can one write $A_{4 m+3}^{+}(t)$ as a sum of two gamma positive summands or show that it cannot be written so? More generally, can we get conditions as to when a polynomial $f(t)$ cannot be written as a sum of two gamma positive polynomials?

For $\pi \in \mathfrak{S}_{n}$, denote ides $(\pi)=\operatorname{des}\left(\pi^{-1}\right)$ and define the two-sided Eulerian polynomial by $\operatorname{TSA}_{n}(s, t)=\sum_{\pi \in \mathfrak{S}_{n}} t^{\operatorname{des}(\pi)+1} s^{\text {ides }(\pi)+1}=\sum_{i, j} a_{n, i, j} t^{i} s^{j}$. The polynomial $\operatorname{TSA}_{n}(s, t)$ is known to satisfy $a_{n, i, j}=a_{n, j, i}$ and $a_{n, i, j}=a_{n, n-i+1, n-j+1}$. Gessel (see [3, Conjecture 10.2]) conjectured that $\operatorname{TSA}_{n}(s, t)=\sum_{i, j} \gamma_{n, i, j}(s t)^{i}(s+t)^{j}(1+s t)^{n+1-2 i-j}$ with $\gamma_{n, i, j} \geqslant 0$. This was recently proved by Lin [11]. See the paper by Adin et al [1] as well.

When $n \equiv 0,1(\bmod 4)$, define $\operatorname{TSA}_{n}^{+}(s, t)=\sum_{\pi \in \mathcal{A}_{n}} t^{\operatorname{des}(\pi)+1} s^{\operatorname{ides}(\pi)+1}=\sum_{i, j} a_{n, i, j}^{+} t^{i} s^{j}$ and likewise define $\operatorname{TSA}_{n}^{-}(s, t)=\sum_{\pi \in \mathfrak{S}_{n}-\mathcal{A}_{n}} t^{\operatorname{des}(\pi)+1} s^{\text {ides }(\pi)+1}=\sum_{i, j} a_{n, i, j}^{-} t^{i} s^{j}$. 
Similarly, when we sum over $\mathfrak{B}_{n}^{+}$and $\mathfrak{B}_{n}^{-}$, define $\operatorname{TSB}_{n}^{+}(s, t)=\sum_{\pi \in \mathfrak{B}_{n}^{+}} t^{\operatorname{des}_{B}(\pi)} s^{\text {ides }_{B}(\pi)}=$ $\sum_{i, j} b_{n, i, j}^{+} t^{i} s^{j}$ and $\operatorname{TSB}_{n}^{-}(s, t)=\sum_{\pi \in \mathfrak{B}_{n}^{-}} t^{\operatorname{des}_{B}(\pi)} s^{\text {ides }_{B}(\pi)}=\sum_{i, j} b_{n, i, j}^{-} t^{i} s^{j}$.

Similarly, when we sum over $\mathfrak{D}_{n}^{+}$and $\mathfrak{D}_{n}^{-}$, define $\operatorname{TSD}_{n}^{+}(s, t)=\sum \sum_{\pi \in \mathfrak{D}_{n}^{+}} t^{\operatorname{des}_{D}(\pi)} s^{\operatorname{ides}_{D}(\pi)}=$ $\sum_{i, j} d_{n, i, j}^{+} t^{i} s^{j}$ and $\operatorname{TSD}_{n}^{-}(s, t)=\sum_{\pi \in \mathfrak{D}_{n}^{-}} t^{\operatorname{des}_{D}(\pi)} s^{\text {ides }_{D}(\pi)}=\sum_{i, j} d_{n, i, j}^{-} t^{i} s^{j}$.

Based on data obtained by using a computer, we make the following.

Conjecture 47. 1. When $n \equiv 0,1(\bmod 4)$, both $\operatorname{TSA}_{n}^{+}(s, t)$ and $\operatorname{TSA}_{n}^{-}(s, t)$ can be written as $\operatorname{TSA}_{n}^{+}(s, t)=\sum_{i, j} \gamma_{n, i, j}^{+}(s t)^{i}(s+t)^{j}(1+s t)^{n+1-2 i-j}$ and $\operatorname{TSA}_{n}^{-}(s, t)=$ $\sum_{i, j} \gamma_{n, i, j}^{-}(s t)^{i}(s+t)^{j}(1+s t)^{n+1-2 i-j}$ with all $\gamma_{n, i, j}^{+}, \gamma_{n, i, j}^{-} \geqslant 0$.

2. When $n \equiv 0(\bmod 2)$, both polynomials $\operatorname{TSB}_{n}^{+}(s, t)$ and $\operatorname{TSB}_{n}^{-}(s, t)$ can be written as $\operatorname{TSB}_{n}^{+}(s, t)=\sum_{i, j} \gamma_{n, i, j}^{B,+}(s t)^{i}(s+t)^{j}(1+s t)^{n-2 i-j}$ and $\operatorname{TSB}_{n}^{-}(s, t)=\sum_{i, j} \gamma_{n, i, j}^{B,-}(s t)^{i}(s+$ $t)^{j}(1+s t)^{n-2 i-j}$ with all $\gamma_{n, i, j}^{B,+}, \gamma_{n, i, j}^{B,-} \geqslant 0$.

3. For integers $n \geqslant 4$, both polynomials $\operatorname{TSD}_{n}^{+}(s, t)$ and $\operatorname{TSD}_{n}^{-}(s, t)$ can be written as $\operatorname{TSD}_{n}^{+}(s, t)=\sum_{i, j} \gamma_{n, i, j}^{D,+}(s t)^{i}(s+t)^{j}(1+s t)^{n-2 i-j}$ and $\operatorname{TSD}_{n}^{-}(s, t)=\sum_{i, j} \gamma_{n, i, j}^{D,-}(s t)^{i}(s+$ $t)^{j}(1+s t)^{n-2 i-j}$ with all $\gamma_{n, i, j}^{D,+}, \gamma_{n, i, j}^{D,-} \geqslant 0$.

It is well known that if a univariate polynomial $f(t)$ is palindromic, has positive coefficients and is real rooted, then it is $\gamma$-positive (see [15, Chapter 4]). Several polynomials that occur in this paper seem to be real rooted. Based on data generated using a computer, we make the following.

Conjecture 48. When $n \geqslant 4$ with $n \equiv 0,1(\bmod 4)$, the polynomials $A_{n}^{+}(t)$ and $A_{n}^{-}(t)$ are real rooted. When $n \geqslant 2$, with $n \equiv 0(\bmod 2)$, the polynomials $B_{n}^{+}(t)$ and $B_{n}^{-}(t)$ are real rooted. When $n \geqslant 3$, the polynomials $D_{n}^{+}(t)$ and $D_{n}^{-}(t)$ are real rooted.

\section{Acknowledgements}

The first author acknowledges support from a CSIR-SPM Fellowship.

The second author acknowledges support from project grant P07 IR052, given by IRCC, IIT Bombay and from project SERB/F/252/2019-2020 given by the Science and Engineering Research Board (SERB), India.

The authors thank the referees for questions which resulted in Section 9 being added to this paper.

\section{References}

[1] Adin, Ron, M., Bagno, E., Eisenberg, E., Reches, S., and Sigron, M. On Two-Sided Gamma-Positivity for Simple Permutations. Electronic Journal of Combinatorics 25(2) (2018), \#P38.

[2] Athanasiadis, C. A. Gamma-positivity in combinatorics and geometry. Seminaire Lotharingien Combin 77 (2016-2018), Art B77i, 64 pp. 
[3] BRÄndÉn, P, M. Actions on permutations and unimodality of descent polynomials. European Journal of Combinatorics 29 (2) (2008), 514-534.

[4] Brändén, P. Unimodality, Log-concavity, Real-rootedness and Beyond. In Handbook of Enumerative Combinatorics, M. Bona, Ed. Chapman \& Hall CRC Press, 2015, ch. 7 .

[5] CHow, C.-O. On certain combinatorial expansions of the Eulerian polynomials. Advances in Applied Math 41 (2008), 133-157.

[6] Foata, D., And DÉsarménien, J. The signed Eulerian numbers. Discrete Mathematics 99 (1992), 49-58.

[7] Fonta, D., And Schützenberger, M.-P. Théorie géométrique des polynômes Eulériens, available at http://www.mat.univie.ac.at/ slc/books/foaschuetz. html. Lecture Notes in Mathematics, 138, Berlin, Springer-Verlag, 1970.

[8] Foata, D., And Strehl, V. Euler numbers and variations of permutations. Colloquio Internazionale sulle Teorie Combinatoire (Roma 1973) Tomo I Atti dei Convegni Lincei, No 17, Accad. Naz. Lincei, Rome (1976), 119-131.

[9] Graham, R. L., Knuth, D. E., And Patashnik, O. Concrete Mathematics, 2nd ed. Pearson Education Asia Pvt Ltd, 2000.

[10] Hyatt, M. Recurrences for Eulerian Polynomials of Type B and Type D. Annals of Combinatorics 20, 4 (2016), 869-881.

[11] Lin, Z. Proof of Gessel's $\gamma$-Positivity Conjecture. Electronic Journal of Combinatorics 23(3) (2016), \#P15.

[12] Ma, S.-M., MA, J., AND YeH, Y.-N. Alternatingly increasing property and bigamma-positivity of polynomials. arXiv:1907.13082 (2019).

[13] Macmahon, P. A. Combinatory Analysis. Cambridge University Press, 1915-1916 (Reprinted by AMS Chelsea, 2000).

[14] Petersen, K. Enriched P-partitions and peak algebras. Advances in Math 209 (2007), 561-610.

[15] Petersen, T. K. Eulerian Numbers, 1st ed. Birkhäuser, 2015.

[16] Reiner, V. Descents and one-dimensional characters for classical Weyl groups. Discrete Mathematics 140 (1995), 129-140.

[17] Shapiro, L. W., Woan, W. J., And Getu, S. Runs, slides and moments. SIAM J. Algebraic Discrete Methods 4(4) (1983), 459-466.

[18] Shin, H., AND ZENG, J. The symmetric and unimodal expansion of Eulerian polynomials via continued fractions. European Journal of Combinatorics 33 (2012), 111-127.

[19] Shin, H., And Zeng, J. Symmetric unimodal expansions of excedances in colored permutations. European Journal of Combinatorics 52 (2016), 174-196.

[20] Sivasubramanian, S. Signed Excedance Enumeration in the Hyperoctahedral group. Electronic Journal of Combinatorics 21(2) (2014), \#P2.10. 
[21] Stembridge, J. Coxeter cones and their h-vectors. Advances in Math 5 (2008), 217.

[22] Tanimoto, S. A Study of Eulerian numbers for Permutations in the Alternating Group. Integers: Electronic Journal of Combinatorial Number Theory 6 (2006), \#A31.

[23] Visontai, M. Some remarks on the joint distribution of descents and inverse descents. Electronic Journal of Combinatorics 20(1) (2013), \#P52. 